\title{
Stability and Bifurcation for a Delayed Diffusive Two-Zooplankton One-Phytoplankton Model with Two Different Functions
}

\author{
Xin-You Meng $(\mathbb{D})$ and Li Xiao \\ School of Science, Lanzhou University of Technology, Lanzhou, Gansu 730050, China \\ Correspondence should be addressed to Xin-You Meng; xymeng@lut.edu.cn
}

Received 25 February 2021; Accepted 27 May 2021; Published 17 June 2021

Academic Editor: Toshikazu Kuniya

Copyright ( 2021 Xin-You Meng and Li Xiao. This is an open access article distributed under the Creative Commons Attribution License, which permits unrestricted use, distribution, and reproduction in any medium, provided the original work is properly cited.

\begin{abstract}
In this paper, a diffusion two-phytoplankton one-zooplankton model with time delay, Beddington-DeAnglis functional response, and Holling II functional response is proposed. First, the existence and local stability of all equilibria of such model are studied. Then, the existence of Hopf bifurcation of the corresponding model without diffusion is given by taking time delay as the bifurcation parameter. Next, the direction of Hopf bifurcation and the stability of the bifurcating periodic solutions are investigated by using the normal form theory and center manifold theorem. Furthermore, due to the local bifurcation theory of partial functional differential equations, Hopf bifurcation of the model is investigated by considering time delay as the bifurcation parameter. The explicit formulas to determine the properties of Hopf bifurcation are given by the method of the normal form theory and center manifold theorem for partial functional differential equations. Finally, some numerical simulations are performed to check out our theoretical results.
\end{abstract}

\section{Introduction}

Generally speaking, plankton is composed of phytoplankton and zooplankton. Phytoplankton is a main producer in the marine ecosystem and the basic link of the food chain. Zooplankton is multiplying, large, and widely distributed. The zooplankton controls the number of phytoplankton by predation and plays an important regulatory role in marine ecosystems.

Plankton systems play an important role in the marine ecosystem, which have always attracted attention of ecologists and mathematicians [1-3]. Many researchers have established a lot of mathematical models to study the dynamic behaviors of plankton systems [4-10]. Phytoplankton can be divided into nontoxic phytoplankton and toxic phytoplankton (TPP). Chattopadhyay et al. [11, 12] showed that toxic phytoplankton and the toxins it released affect the growth rate of zooplankton populations and the interaction between phytoplankton and zooplankton. Chattopadhyay et al. [13] proposed a delayed differential equation for toxins and pointed out that toxic phytoplankton or toxic substances may be the key reason for the termination of plankton blooms. Similar results can be found in references [14-19]. In their work [15], Saha and Bandyopadhyay considered the following TPP-zooplankton model:

$$
\left\{\begin{array}{l}
\frac{\mathrm{d} P(t)}{\mathrm{d} t}=r P(t)\left(1-\frac{P(t)}{K}\right)-\frac{\mu P(t) Z(t)}{\alpha+P(t)}, \\
\frac{\mathrm{d} Z(t)}{\mathrm{d} t}=\frac{\beta_{1} P(t) Z(t)}{\alpha+P(t)}-\delta Z(t)-\frac{\rho P(t-\tau) Z(t)}{\alpha+P(t-\tau)},
\end{array}\right.
$$

where $P(t)$ and $Z(t)$ represent the density of TPP and zooplankton, respectively, $r$ and $K$ denote the intrinsic growth rate and environmental capacity of TPP, $\mu$ is the maximum predation rate of zooplankton, $\beta_{1}$ is the ratio of biomass consumed per zooplankton for the production of new zooplankton $\left(0<\beta_{1}<1\right), \delta$ is the natural death rate of 
zooplankton, $\alpha$ denotes the half saturation constant, $\rho$ is the ratio of toxic substances produced per unit biomass of TPP, and $\tau$ represents the discrete time required for maturation of TPP to release toxic substances. They [15] found that system (1) is locally asymptotically stable at the positive equilibrium when $\tau$ is less than its threshold; otherwise, system (1) undergoes Hopf bifurcation.
There are many types of zooplankton in marine ecology and freshwater biological systems. Each zooplankton has its own living habit. There is an inseparable relationship among them. Therefore, the impact of different species on the ecosystem is the concern for most scholars. Some researchers considered two types of zooplankton [20-24]. Lv et al. [22] investigated the complex dynamics of the two zooplankton-phytoplankton model with delay:

$$
\left\{\begin{array}{l}
\frac{\mathrm{d} P(t)}{\mathrm{d} t}=r P(t)\left(1-\frac{P(t)}{K}\right)-\frac{\mu_{1} P(t) Z_{1}(t)}{\alpha_{1}+P(t)}-\frac{\mu_{2} P(t) Z_{2}(t)}{\alpha_{2}+P(t)} \\
\frac{\mathrm{d} Z_{1}(t)}{\mathrm{d} t}=\frac{\beta_{1} P(t) Z_{1}(t)}{\alpha_{1}+P(t)}-\frac{\rho_{1} P(t-\tau) Z_{1}(t)}{\alpha_{1}+P(t-\tau)}-d_{1} Z_{1}(t)-g_{1} Z_{1}^{2}(t), \\
\frac{\mathrm{d} Z_{2}(t)}{\mathrm{d} t}=\frac{\beta_{2} P(t) Z_{2}(t)}{\alpha_{2}+P(t)}-\frac{\rho_{2} P(t-\tau) Z_{2}(t)}{\alpha_{2}+P(t-\tau)}-d_{2} Z_{2}(t)-g_{2} Z_{2}^{2}(t),
\end{array}\right.
$$

where $P(t), Z_{1}(t)$, and $Z_{2}(t)$ represent the density of TPP, zooplankton 1 , and zooplankton 2 at time $t$, respectively. The parameter $\mu_{i}$ is the maximum uptake rate of zooplankton $i(i=1$ and 2$)$. $\beta_{i}$ denotes the ratio of biomass conversion of zooplankton $i(i=1$ and 2$) . \rho_{i}$ is the ratio of toxic substances produced by per unit biomass of TPP for the zooplankton $i(i=1$ and 2$) . \alpha_{i}$ represents the half saturation constant of zooplankton $i(i=1$ and 2$) . g_{i}$ is the interspecific competition coefficient of zooplankton $i(i=1$ and 2$) . d_{i}$ denotes the natural mortality of corresponding zooplankton $i(i=1$ and 2$)$. The biological significance of other parameters is the same as system (1). All parameters are positive constants. System (2) shows that the intrinsic characteristics of toxins, such as the release rate of toxicity and the delay of toxin production, does not irreversibly change the stability of this system. It is finally concluded that toxin-producing phytoplankton may be used as a biocontrol agent for the harmful algal bloom problems.

In nature, species interact with each other to produce different biological effects. Each effect can vividly describe the relationship between species. Beddington [25] and DeAngelis et al. [26] proposed Beddington-DeAnglis functional response (B-D function), which is similar to the functional response of the famous Holling type II. The B-D function can better reflect the prey-dependent and the mutual interference between predators [27-33]. Cantrell and Cosner [27] investigated the predator-prey model with the B-D function and showed that the degree of mutual interference between predators affects the position and stability of the equilibria. Meng and Wang [31] established a delayed diffusive model with the B-D function and discussed the existence of Hopf bifurcation and its properties.
From a biological point of view, individual organisms are distributed in space, usually interacting with the physical environment and other organisms in their spatial neighborhood. Generally speaking, self-diffusion refers to the transmission from an area of higher concentration to a low concentration area through random motion. The diffusion of individuals may be related to other things, such as finding food and escaping from high risk of infection. Hardy [34] pointed out that the spatial distribution of marine plankton is not uniform. The cooperativity of plankton is weak, but plankton can move freely under the influence of ocean currents and monsoons. This spatial diffusion is subject to Fick's law. Therefore, the influence of spatial diffusion on the phytoplankton-plankton model has been paid more attention by many scholars [35-45]. In the work by Jia et al. [46], a three-component plankton model with spatial diffusion and time delay is proposed, which describes the relationship between a zooplankton and two phytoplankton. Rao [47] studied the complex dynamics of a spatial toxicphytoplankton-zooplankton model with the Holling II function and showed that the interaction between toxic phytoplankton and zooplankton in the marine environment may be partially driven by diffusivity or environmental carrying capacity.

Encouraged by the above work, we will consider the Beddington-DeAngelis functional response and the effect of self-diffusion for phytoplankton and zooplankton into system (2) in this paper. Thus, a diffusion toxic phytoplankton-zooplankton model with time delay and two kinds of functional responses is given as follows: 


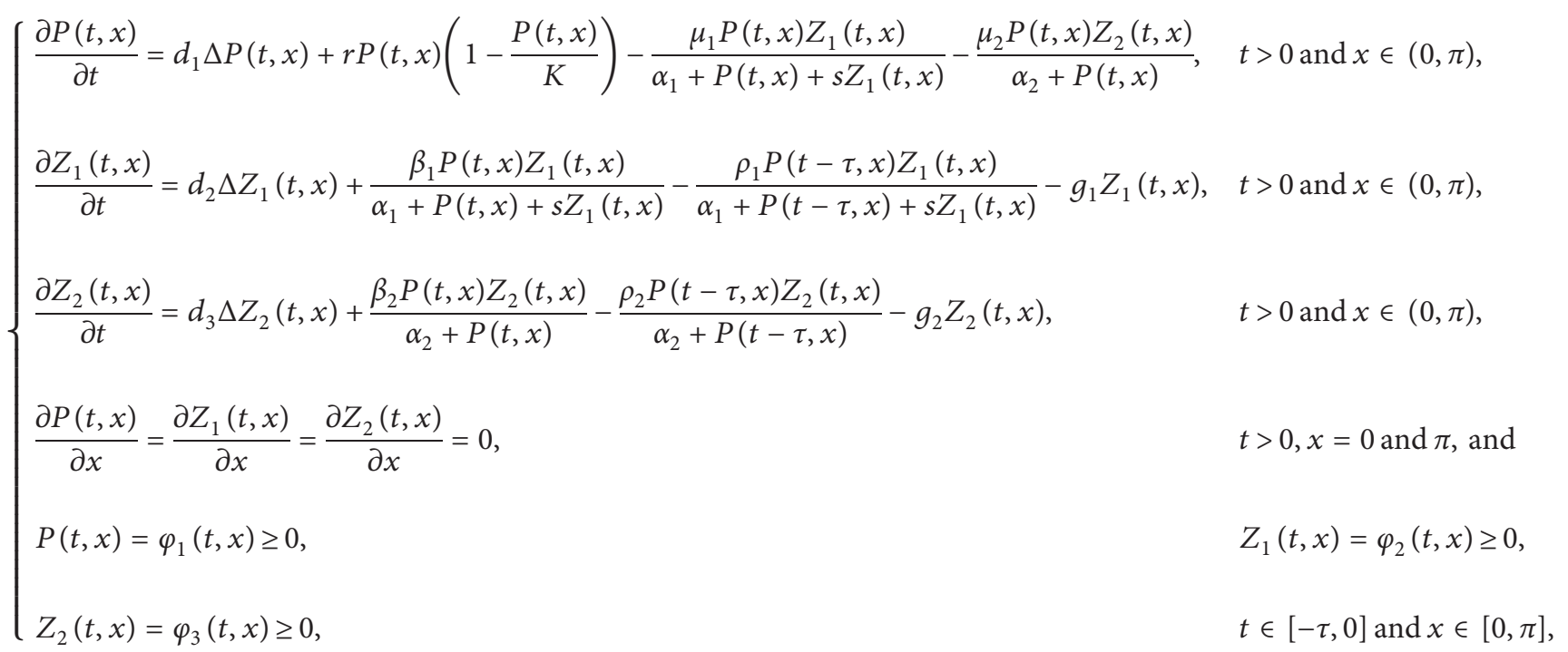

where $P(t, x), Z_{1}(t, x)$, and $Z_{2}(t, x)$ represent the densities of the toxic-phytoplankton and two kinds of zooplankton at location $x$ and time $t$, respectively. $\Delta$ is the Laplace operator, and the homogeneous Neumann boundary condition means that no plankton species enters or leaves this area. $d_{1}, d_{2}$, and $d_{3}$ are the diffusion rates of the three species, $g_{i}$ is expressed as natural mortality of zooplankton $i(i=1$ and 2$)$, and $s$ is the measure of the degree of mutual interference among zooplankton $Z_{1}$. The remaining parameters have the same biological meanings as model (2). All parameters are positive constants.

The remain parts of this paper are organized as follows. The existence and stability of equilibria are discussed in Section 2. The existence and properties of Hopf bifurcation of model (2) with time delay by the normal form theory and the center manifold theorem are given in Section 3. In Section 4, we analyze the Hopf bifurcation of model (3) at the coexistence equilibrium and give the explicit formulas to determine the properties of Hopf bifurcation by using the normal form theory and center manifold theorem for partial functional differential equations (PFDEs). In Section 5, numerical simulations are given to verify the theoretical results. A discussion is given to conclude this work in Section 6.

\section{Existence and Stability of Equilibria of ODE}

In this section, we investigate the dynamic behavior of system (3) without delay and diffusion. The corresponding ordinary differential model of system (3) is as follows:

$$
\left\{\begin{array}{l}
\frac{\mathrm{d} P(t)}{\mathrm{d} t}=r P(t)\left(1-\frac{P(t)}{K}\right)-\frac{\mu_{1} P(t) Z_{1}(t)}{\alpha_{1}+P(t)+s Z_{1}(t)}-\frac{\mu_{2} P(t) Z_{2}(t)}{\alpha_{2}+P(t)} \\
\frac{\mathrm{d} Z_{1}(t)}{\mathrm{d} t}=\frac{\beta_{1} P(t) Z_{1}(t)}{\alpha_{1}+P(t)+s Z_{1}(t)}-\frac{\rho_{1} P(t) Z_{1}(t)}{\alpha_{1}+P(t)+s Z_{1}(t)}-g_{1} Z_{1}(t) \\
\frac{\mathrm{d} Z_{2}(t)}{\mathrm{d} t}=\frac{\beta_{2} P(t) Z_{2}(t)}{\alpha_{2}+P(t)}-\frac{\rho_{2} P(t) Z_{2}(t)}{\alpha_{2}+P(t)}-g_{2} Z_{2}(t)
\end{array}\right.
$$

The Jacobian matrix of system (4) is 


$$
J=\left(\begin{array}{ccc}
\frac{K r-2 r P}{K}-\frac{\mu_{1} Z_{1}\left(\alpha_{1}+s Z_{1}\right)}{\left(\alpha_{1}+P+s Z_{1}\right)^{2}}-\frac{\mu_{2} Z_{2} \alpha_{2}}{\left(\alpha_{2}+P\right)^{2}} & -\frac{\mu_{1} P\left(\alpha_{1}+P\right)}{\left(\alpha_{1}+P+s Z_{1}\right)^{2}} & -\frac{\mu_{2} P}{\alpha_{2}+P} \\
\frac{\left(\beta_{1}-\rho_{1}\right) Z_{1}\left(\alpha_{1}+s Z_{1}\right)}{\left(\alpha_{1}+P+s Z_{1}\right)^{2}} & \frac{\left(\beta_{1}-\rho_{1}\right) P\left(\alpha_{1}+P\right)}{\left(\alpha_{1}+P+s Z_{1}\right)^{2}}-g_{1} & 0 \\
\frac{\left(\beta_{2}-\rho_{2}\right) Z_{2} \alpha_{2}}{\left(\alpha_{2}+P\right)^{2}} & 0 & \frac{\left(\beta_{2}-\rho_{2}\right) P}{\alpha_{2}+P}-g_{2}
\end{array}\right) .
$$

2.1. The Trivial Equilibriums. It is obvious that system (4) has the trivial equilibrium $E_{0}(0,0,0)$ and the semitrivial equilibrium $E_{1}(K, 0,0)$. $E_{0}$ is

The characteristic equation of system (4) at equilibrium

$$
(\lambda-r)\left(\lambda+g_{1}\right)\left(\lambda+g_{2}\right)=0
$$

Therefore, we can obtain three eigenvalues:

$$
\begin{aligned}
& \lambda_{1}=r>0, \\
& \lambda_{2}=-g_{1}<0, \\
& \lambda_{3}=-g_{2}<0 .
\end{aligned}
$$

Thus, the trivial equilibrium $E_{0}$ is unstable.

The local stability of system (4) at the semitrivial equilibrium $E_{1}(K, 0,0)$ is as follows.

We can obtain that the characteristic equation of system (4) at the semitrivial equilibrium $E_{1}$ is as follows:

$$
(\lambda+r)\left[\lambda-\left(\frac{\left(\beta_{1}-\rho_{1}\right) K}{\alpha_{1}+K}-g_{1}\right)\right]\left[\lambda-\left(\frac{\left(\beta_{2}-\rho_{2}\right) K}{\alpha_{2}+K}-g_{2}\right)\right]=0 \text {. }
$$

From equation (8), we can get three eigenvalues:

$$
\begin{aligned}
& \lambda_{1}=-r<0, \\
& \lambda_{2}=\frac{\left(\beta_{1}-\rho_{1}\right) K}{\alpha_{1}+K}-g_{1}, \\
& \lambda_{3}=\frac{\left(\beta_{2}-\rho_{2}\right) K}{\alpha_{2}+K}-g_{2} .
\end{aligned}
$$

So $\lambda_{2}<0$ and $\lambda_{3}<0$ if and only if $K<$ $\min \left\{\left(g_{1} \alpha_{1} /\left(\beta_{1}-\rho_{1}-g_{1}\right)\right),\left(g_{2} \alpha_{2} /\left(\beta_{2}-\rho_{2}-g_{2}\right)\right)\right\}$. Thus, the semitrivial equilibrium $E_{1}$ is locally asymptotically stable; otherwise, $E_{1}$ is unstable.

Theorem 1. System (4) is unstable at the trivial equilibrium $E_{0}$. System (4) is locally asymptotically stable at the semitrivial equilibrium $E_{1}$ if $K<\min \left\{\left(g_{1} \alpha_{1} /\left(\beta_{1}-\rho_{1}-g_{1}\right)\right)\right.$, $\left.\left(g_{2} \alpha_{2} /\left(\beta_{2}-\rho_{2}-g_{2}\right)\right)\right\}$, but system (4) is unstable if $K \geq \min \left\{\left(g_{1} \alpha_{1} /\left(\beta_{1}-\rho_{1}-g_{1}\right)\right),\left(g_{2} \alpha_{2} /\left(\beta_{2}-\rho_{2}-g_{2}\right)\right)\right\}$.

2.2. The Boundary Equilibriums. System (4) has two boundary equilibriums. From the second equation of system
(4), we can obtain that $Z_{1}=\left(1 / g_{1} s\right)\left[\left(\beta_{1}-\rho_{1}-g_{1}\right) P-g_{1} \alpha_{1}\right]$. Then, by substituting it into the first equation of system (4), we can obtain that the following quadratic equation

$$
M_{1} P^{2}+M_{2} P+M_{3}=0
$$

where $M_{1}=r s \quad\left(\beta_{1}-\rho_{1}\right), M_{2}=K \mu_{1}\left(\beta_{1}-\rho_{1}-g_{1}\right)-r s K$ $\left(\beta_{1}-\rho_{1}\right)$, and $M_{3}=-\mu_{1} K g_{1} \alpha_{1}$.

If the assumption $\beta_{1}-\rho_{1}-g_{1}>0$, then $M_{1}>0$ and $\Delta_{1}=M_{2}^{2}-4 M_{1} M_{3}>0$; thus, equation (10) has at least one positive root, defined as $\bar{P}=\left(\left(-M_{2}\right.\right.$

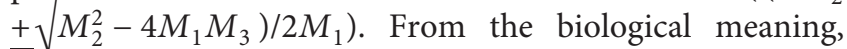
$\bar{Z}_{1}=\left(1 / g_{1} s\right)\left[\left(\beta_{1}-\rho_{1}-g_{1}\right) \bar{P}-g_{1} \alpha_{1}\right]$ is nonnegative when $\left(\beta_{1}-\rho_{1}-g_{1}\right) \bar{P}>g_{1} \alpha_{1}$. If the assumption $\left(H_{1}\right): \beta_{1}-\rho_{1}-g_{1}>0,\left(\beta_{1}-\rho_{1}-g_{1}\right) \bar{P}>g_{1} \alpha_{1}$ holds, then system (4) has the boundary equilibrium $E_{10}\left(\bar{P}, \bar{Z}_{1}, 0\right)$.

Now, under the assumption $\left(H_{1}\right)$, we prove the stability of the boundary equilibrium $E_{10}$. Linearizing system (4) at $E_{10}$, we get the characteristic equation:

$$
\left(\lambda-A_{33}\right)\left[\lambda^{2}-\left(A_{11}+A_{22}\right) \lambda+A_{11} A_{22}-A_{12} A_{21}\right]=0,
$$

where

$$
\begin{aligned}
& A_{11}=\frac{\mu_{1} g_{1}^{2} \bar{Z}_{1}-\frac{r \bar{P}}{\left(\beta_{1}-\rho_{1}\right)^{2} \bar{P}},}{A_{22}=}=\frac{g_{1}\left[g_{1} \alpha_{1}+\bar{P}\left(g_{1}+\rho_{1}-\beta_{1}\right)\right]}{\left(\beta_{1}-\rho_{1}\right) \bar{P}}, \\
& A_{13}=-\frac{\mu_{2} \bar{P}}{\left(\alpha_{2}+\bar{P}\right)^{2}}, \\
& A_{21}=\frac{\left(\beta_{1}-\rho_{1}\right) \bar{Z}_{1}\left(\alpha_{1}+s \bar{Z}_{1}\right)}{\left(\alpha_{1}+\bar{P}+s \bar{Z}\right)^{2}}, \\
& A_{12}=-\frac{\mu_{1} \bar{P}\left(\alpha_{1}+\bar{P}\right)}{\left(\alpha_{1}+\bar{P}+s \bar{Z}\right)^{2}}, \\
& A_{33}=\frac{\left(\beta_{2}-\rho_{2}-g_{2}\right) \bar{P}-g_{2} \alpha_{2}}{\alpha_{2}+\bar{P}} .
\end{aligned}
$$

Further $\lambda_{1}=A_{33}, \lambda_{2}$ and $\lambda_{3}$ are the roots of the quadratic equation:

$$
\lambda^{2}+M_{4} \lambda+M_{5}=0
$$


where $M_{4}=-\left(A_{11}+A_{22}\right)$ and $M_{5}=A_{11} A_{22}-A_{12} A_{21}=0$. Let $\Delta_{2}=M_{4}^{2}-4 M_{5}$; we assume that $\left(H_{2}\right)$ : $\Delta_{2}=M_{4}^{2}-4 M_{5} \geq 0, \quad\left(\mu_{1} \bar{Z}_{1} /\left(\alpha_{1}+\bar{P}+s \bar{Z}_{1}\right)^{2}\right)<(r / K)$, and $\bar{P}<\left(g_{2} \alpha_{2} /\left(\beta_{2}-\rho_{2}-g_{2}\right)\right)$. Under the assumption $\left(H_{2}\right)$, $A_{33}<0, M_{4}>0$, and $M_{5}>0$. Thus, equation (13) has two negative roots. Then, we have the following conclusion.

Theorem 2. The boundary equilibrium $E_{10}$ exists if $\left(H_{1}\right)$ holds. Furthermore, $E_{10}$ of system (4) is locally asymptotically stable if assumptions $\left(H_{1}\right)$ and $\left(H_{2}\right)$ hold.

Similarly, the other boundary equilibrium $E_{01}\left(\widetilde{P}, 0, \widetilde{Z}_{2}\right)$ exists when the assumption $\left(H_{3}\right)$ : $\beta_{2}-\rho_{2}-g_{2}>0$ and $K\left(\beta_{2}-\rho_{2}-g_{2}\right)>g_{2} \alpha_{2}$ holds, where $\widetilde{P}=$ $\left(g_{2} \alpha_{2} /\left(\beta_{2}-\rho_{2}-g_{2}\right)\right)$ and $Z_{2}=\left(\left(r \alpha_{2}\left[K\left(\beta_{2}-\rho_{2}-g_{2}\right)-\right.\right.\right.$ $\left.\left.\left.g_{2} \alpha_{2}\right]\left(\beta_{2}-\rho_{2}\right)\right) / \mu_{2} K\left(\beta_{2}-\rho_{2}-g_{2}\right)^{2}\right)$.

Next, we investigate stability of the boundary equilibrium $E_{01}$. The method is the same to that of the boundary equilibrium $E_{10}$.

The characteristic equation of system (4) at the boundary equilibrium $E_{01}$ is

$$
\left(\lambda-B_{22}\right)\left(\lambda^{2}-B_{11} \lambda-B_{13} B_{31}\right)=0,
$$

where

$$
\begin{aligned}
& B_{11}=\frac{\mu_{2} \widetilde{P} \widetilde{Z}_{2}}{\left(\alpha_{2}+\widetilde{P}\right)^{2}}-\frac{r}{K}, \\
& B_{22}=\frac{\left(\beta_{1}-\rho_{1}-g_{1}\right) \widetilde{P}-g_{1} \alpha_{1}}{\alpha_{1}+\widetilde{P}}, \\
& B_{13}=-\frac{\mu_{2} \widetilde{P}}{\alpha_{2}+\widetilde{P}}<0, \\
& B_{31}=\frac{\left(\beta_{2}-\rho_{2}\right) \alpha_{2} \widetilde{Z}_{2}}{\left(\alpha_{1}+\widetilde{P}\right)^{2}}>0 .
\end{aligned}
$$

If $\lambda_{1}=B_{22}$, then $\lambda_{2}$ and $\lambda_{3}$ are the roots of the following equation:

$$
\lambda^{2}-B_{11} \lambda-B_{13} B_{31}=0 .
$$

Let $\Delta_{3}=B_{11}^{2}+4 B_{13} B_{31}$; we assume that $\left(H_{4}\right): \Delta_{3}>0$ and $\left(\beta_{2}-\rho_{2}-g_{2}\right)\left(\beta_{2}-\rho_{2}-g_{2}\right) K+g_{2}^{2} \alpha_{2}>0$. Under the assumption $\left(H_{4}\right), \lambda_{1}=B_{22}<0, B_{11}<0$, and $B_{13} B_{31}<0$; thus, equation (16) has two real negative roots. Then, we have the following result.

Theorem 3. If assumption $\left(\mathrm{H}_{3}\right)$ is true, the boundary equilibrium $E_{01}$ of system (4) exists. Further, if assumptions $\left(\mathrm{H}_{3}\right)$ and $\left(\mathrm{H}_{4}\right)$ are true, then the boundary equilibrium $E_{01}$ of system (4) is locally asymptotically stable.

2.3. The Coexistence Equilibrium. In biology, we are interested in the existence and stability of the coexistence equilibrium. Next, we will analyze the existence of the coexistence equilibrium of system (4). System (4) has a coexistence equilibrium $E^{*}\left(P^{*}, Z_{1}^{*}, Z_{2}^{*}\right)$, where $P^{*}, Z_{1}^{*}$, and $Z_{2}^{*}$ are the positive solution of the following equations:

$$
\left\{\begin{array}{l}
r\left(1-\frac{P}{K}\right)-\frac{\mu_{1} Z_{1}}{\alpha_{1}+P+s Z_{1}}-\frac{\mu_{2} Z_{2}}{\alpha_{2}+P}=0, \\
\frac{\left(\beta_{1}-\rho_{1}\right) P}{\alpha_{1}+P+s Z_{1}}-g_{1}=0, \\
\frac{\left(\beta_{2}-\rho_{2}\right) P}{\alpha_{2}+P}-g_{2}=0 .
\end{array}\right.
$$

From the third equation of (17), we can get that $P^{*}=$ $\left(g_{2} \alpha_{2} /\left(\beta_{2}-\rho_{2}-g_{2}\right)\right)$ if $\beta_{2}-\rho_{2}-g_{2}>0$ holds. By substituting $P^{*}$ into the second equation of (17), we can get that $Z_{1}^{*}=\left(K_{1} / g_{1} s\left(\beta_{2}-\rho_{2}-g_{2}\right)\right) \quad$ if $K_{1}>0$, where $K_{1}=g_{2} \alpha_{2}\left(\beta_{1}-\rho_{1}-g_{1}\right)-g_{1} \alpha_{1}\left(\beta_{2}-\rho_{2}-g_{2}\right)$. By substituting $P^{*}$ and $Z_{1}^{*}$ into the first equation of (17), we can get that $Z_{2}^{*}=\left(\left(\alpha_{1}+P^{*}\right) K_{2} /\left(g_{2} \mu_{2} \alpha_{2} K\left(\beta_{1}-\rho_{1}\right)\left(\beta_{2}-\rho_{2}-g_{2}\right)\right)\right) \quad$ if $\left(r-\mu_{1}\right) K\left(\beta_{2}-\rho_{2}-g_{2}\right)-r g_{2} \alpha_{2}>0$ holds, where $K_{2}=g_{2} \alpha_{2}\left(\beta_{1}-\rho_{1}\right)\left[\begin{array}{lll}\left(r-\mu_{1}\right) K & \left(\beta_{2}-\rho_{2}-g_{2}\right) & -r g_{2} \alpha_{2}\end{array}\right]+$ $\mu_{1} g_{1} K\left(\beta_{2}-\rho_{2}-g_{2}\right)\left[g_{2} \alpha_{2}+\alpha_{1}\left(\beta_{2}-\rho_{2}-g_{2}\right)\right]$. Thus, system (4) has one positive equilibrium $E^{*}\left(P^{*}, Z_{1}^{*}, Z_{2}^{*}\right)$ when the condition $\left(H_{5}\right): \beta_{2}>\rho_{2}+g_{2}, K_{1}>0$, and $\left(r-\mu_{1}\right) K\left(\beta_{2}-\rho_{2}-\right.$ $\left.g_{2}\right)-r g_{2} \alpha_{2}>0$ is true.

From a biological point of view, the stable coexistence of multiple species is very meaningful. Now we discuss the stability of the positive equilibrium $E^{*}$. The characteristic equation of system (4) at $E^{*}$ is

$$
\lambda^{3}+M_{6} \lambda^{2}+M_{7} \lambda+M_{8}=0,
$$

where

$$
\begin{aligned}
& C_{11}=\frac{\mu_{1} Z_{1}^{*} P^{*}}{\left(\alpha_{1}+P^{*}+s Z_{1}^{*}\right)^{2}}+\frac{\mu_{2} P^{*} Z_{2}^{*}}{\left(\alpha_{2}+P^{*}\right)^{2}}-\frac{r P^{*}}{K}, \\
& C_{12}=-\frac{\mu_{1} P^{*}\left(\alpha_{1}+P^{*}\right)}{\left(\alpha_{1}+P^{*}+s Z_{1}^{*}\right)^{2}}, \\
& C_{13}=-\frac{\mu_{2} P^{*}}{\alpha_{2}+P^{*}}, \\
& C_{21}=\frac{\left(\beta_{1}-\rho_{1}\right) Z_{1}^{*}\left(\alpha_{1}+s Z_{1}^{*}\right)}{\left(\alpha_{1}+P^{*}+s Z_{1}^{*}\right)^{2}}, \\
& C_{22}=-\frac{s\left(\beta_{1}-\rho_{1}\right) P^{*} Z_{1}^{*}}{\left(\alpha_{1}+P^{*}+s Z_{1}^{*}\right)^{2}}, \\
& C_{31}=\frac{\left(\beta_{2}-\rho_{2}\right) Z_{2}^{*} \alpha_{2}}{\left(\alpha_{2}+P^{*}\right)^{2}}, \\
& M_{6}=-\left(C_{11}+C_{22}\right), \\
& M_{7}=C_{11} C_{22}-C_{12} C_{21}-C_{13} C_{31}, \\
& M_{8}=C_{13} C_{31} C_{22} .
\end{aligned}
$$


By the Routh-Hurwitz criterion [48], $E^{*}$ is locally asymptotically stable if $M_{6}>0, M_{8}>0$, and $M_{6} M_{7}-M_{8}>0$. Hence, we assume that $\left(H_{6}\right):\left(\left(\mu_{1}\left(\beta_{1}-\rho_{1}\right)^{2} g_{2}^{2} \alpha{ }_{2}^{2} K_{1}\right) /\left(g_{1}^{3} s\right.\right.$ $\left.\left.\left(\beta_{2}-\rho_{2}-g_{2}\right)^{3}\right)\right)+\left(\left(\alpha_{2}^{2}\left(\beta_{2}-\rho_{2}\right)^{2} K_{2}\right) /\left(K g_{2}\left(\beta_{1}-\rho_{1}\right)\left(\beta_{2}-\right.\right.\right.$ $\left.\left.\left.\rho_{2}-g_{2}\right)^{3}\right)\right)<(r / K)$. Then, we have the following conclusion.

Theorem 4. Under the assumption $\left(H_{5}\right)$, the coexistence equilibrium $E^{*}$ of system (4) is locally asymptotically stable if the assumption $\left(\mathrm{H}_{6}\right)$ holds.

Now, we consider the parameter $s$ as a bifurcation parameter to study the existence of Hopf bifurcation. According to Liu [49], we can get that the following result.

Theorem 5. If the characteristic equation of the coexistence equilibrium is given by

$$
\lambda^{3}+M_{6}(s) \lambda^{2}+M_{7}(s) \lambda+M_{8}(s)=0,
$$

where $M_{6}(s), \Omega=M_{6}(s) M_{7}(s)-M_{8}(s)$, and $M_{8}(s)$ are smooth function of $s$ in an open interval about $s^{*} \in R$ such that

(1) $M_{6}\left(s^{*}\right)>0, \Omega\left(s^{*}\right)=0$, and $M_{8}\left(s^{*}\right)>0$

(2) $\left.(d \Omega / d s)\right|_{s=s^{*}} \neq 0$; then, a simple Hopf bifurcation occurs at $s=s^{*}$

Proof. In fact, $M_{6}(s)=M_{6}, \Omega(s)=M_{6} M_{7}-M_{8}$, and $M_{8}(s)=M_{8}$. It is easy to see that there exists $s^{*}$ such that $M_{6}\left(s^{*}\right)>0, \quad \Omega\left(s^{*}\right)=0, \quad M_{8}\left(s^{*}\right)>0,\left.\quad\left(\mathrm{~d} P^{*} / \mathrm{d} s\right)\right|_{s=s^{*}}=0$, $\left.\left(\mathrm{d} Z_{1}^{*} / \mathrm{d} s\right)\right|_{s=s^{*}}=-\left(\left(\left(\beta_{1}-\rho_{1}-g_{1}\right) P^{*}-g_{1} \alpha_{1}\right) / g_{1} s^{2}\right) \neq 0$, and $\left.\left(\mathrm{d} Z_{2}^{*} / \mathrm{d} s\right)\right|_{s=s^{*}}=-\left(\left(g_{1} \mu_{1}\left(\beta_{2}-\rho_{2}\right)\right) /\left(\mu_{2} g_{2}\left(\beta_{1}-\rho_{1}\right)\right)\right) \cdot\left(\mathrm{d} Z_{1}^{*} /\right.$ $\mathrm{d} s) \neq 0$. So, we can get $\left.(\mathrm{d} \Omega / \mathrm{d} s)\right|_{s=s^{*}} \neq 0$. Thus, the Hopf bifurcation occurs at $s=s^{*}$.

\section{Hopf Bifurcation of System (3) without Diffusion}

3.1. The Existence of Bifurcation. In this section, under the assumption $\left(\mathrm{H}_{5}\right)$, we regard time delay $\tau$ as the bifurcation parameter to study its influence on the stability of coexistence equilibrium $E^{*}\left(P^{*}, Z_{1}^{*}, Z_{2}^{*}\right)$. System (4) with time delay is as follows:

$$
\left\{\begin{array}{l}
\frac{\mathrm{d} P}{\mathrm{~d} t}=r P\left(1-\frac{P}{K}\right)-\frac{\mu_{1} P Z_{1}}{\alpha_{1}+P+s Z_{1}}-\frac{\mu_{2} P Z_{2}}{\alpha_{2}+P}, \\
\frac{\mathrm{d} Z_{1}}{\mathrm{~d} t}=\frac{\beta_{1} P Z_{1}}{\alpha_{1}+P+s Z_{1}}-\frac{\rho_{1} P(t-\tau) Z_{1}}{\alpha_{1}+P(t-\tau)+s Z_{1}}-g_{1} Z_{1}, \\
\frac{\mathrm{d} Z_{2}}{\mathrm{~d} t}=\frac{\beta_{2} P Z_{2}}{\alpha_{2}+P}-\frac{\rho_{2} P(t-\tau) Z_{2}}{\alpha_{2}+P(t-\tau)}-g_{2} Z_{2} .
\end{array}\right.
$$

Let $u_{1}(t)=P(t)-P^{*}, u_{2}(t)=Z_{1}(t)-Z_{1}^{*}, u_{3}(t)=Z_{2}(t)$ $-Z_{2}^{*}$, and $U(t)=\left(u_{1}(t), u_{2}(t), u_{3}(t)\right)^{T}$. Substituting $U(t)$ into system (21), we can get the following linearized system:

$$
\frac{\mathrm{d} U(t)}{\mathrm{d} t}=L_{1}(U(t))+L_{2}(U(t-\tau))
$$

where

$$
\begin{aligned}
L_{1} & =\left(\begin{array}{lll}
a_{11} & a_{12} & a_{13} \\
a_{21} & a_{22} & 0 \\
a_{31} & 0 & 0
\end{array}\right), \\
L_{2} & =\left(\begin{array}{lll}
0 & 0 & 0 \\
b_{21} & 0 & 0 \\
b_{31} & 0 & 0
\end{array}\right),
\end{aligned}
$$

where $a_{1 k}=C_{1 k}(k=1,2,3)$ and

$$
\begin{aligned}
& a_{21}=\frac{\beta_{1} Z_{1}^{*}\left(\alpha_{1}+s Z_{1}^{*}\right)}{\left(\alpha_{1}+P^{*}+s Z_{1}^{*}\right)^{2}}, \\
& a_{22}=\frac{\left(\beta_{1}-\rho_{1}\right) P^{*}\left(\alpha_{1}+P^{*}\right)}{\left(\alpha_{1}+P^{*}+s Z_{1}^{*}\right)^{2}}-g_{1}, \\
& a_{31}=\frac{\beta_{2} Z_{2}^{*} \alpha_{2}}{\left(\alpha_{2}+P^{*}\right)^{2}}, \\
& b_{21}=-\frac{\rho_{1} Z_{1}^{*}\left(\alpha_{1}+s Z_{1}^{*}\right)}{\left(\alpha_{1}+\bar{P}+s Z_{1}^{*}\right)^{2}}, \\
& b_{31}=-\frac{\rho_{2} Z_{2}^{*} \alpha_{2}}{\left(\alpha_{2}+P^{*}\right)^{2}} .
\end{aligned}
$$

Therefore, the characteristic equation of system (22) is

$$
\lambda^{3}+D_{2} \lambda^{2}+D_{1} \lambda+D_{0}+e^{-\lambda \tau}\left(F_{1} \lambda+F_{0}\right)=0,
$$

where

$$
\begin{aligned}
& D_{2}=-\left(a_{11}+a_{22}\right), \\
& D_{1}=a_{11} a_{22}-a_{12} a_{21}-a_{13} a_{31}, \\
& D_{0}=a_{13} a_{22} a_{31}, \\
& F_{1}=-\left(a_{13} b_{31}+a_{12} b_{21}\right), \\
& F_{0}=a_{13} a_{22} b_{31} .
\end{aligned}
$$

When $\tau=0$, the distribution of roots of equation (25) has been discussed above. Next, we will discuss the distribution of characteristic roots of equation (25) when $\tau>0$. First, we suppose that $i \omega_{1}\left(\omega_{1}>0\right)$ is a root of equation (25). Then, we obtain that

$-i \omega_{1}^{3}-D_{2} \omega_{1}^{2}+i D_{1} \omega_{1}+D_{0}+\left(\cos \omega_{1} \tau-i \sin \omega_{1} \tau\right)\left(i F_{1} \omega_{1}-F_{0}\right)=0$.

Separating the real and imaginary parts of equation (27), we have

$$
\left\{\begin{array}{l}
\omega_{1}^{3}-D_{1} \omega_{1}=F_{1} \omega_{1} \cos \omega_{1} \tau-F_{0} \sin \omega_{1} \tau \\
D_{2} \omega_{1}^{2}-D_{0}=F_{0} \cos \omega_{1} \tau+F_{1} \omega_{1} \sin \omega_{1} \tau .
\end{array}\right.
$$

From equation (28), we get that 
$\omega_{1}^{6}+\left(D_{2}^{2}-2 D_{1}\right) \omega_{1}^{4}+\left(D_{1}^{2}-2 D_{0} D_{2}-F_{1}^{2}\right) \omega_{1}^{2}+D_{0}^{2}-F_{0}^{2}=0$

Let $\omega_{1}^{2}=y, M_{9}=D_{2}^{2}-2 D_{1}, M_{10}=D_{1}^{2}-2 D_{0} D_{2}-F_{1}^{2}$, and $M_{11}=D_{0}^{2}-F_{0}^{2}$. Then, equation (29) can be rewritten as

$$
f(y) \triangleq y^{3}+M_{9} y^{2}+M_{10} y+M_{11}=0 .
$$

To obtain the existence of Hopf bifurcation, equation (30) has at least one positive root. Due to $f(0)=$ $M_{11}=a_{13}^{2} a_{22}^{2}\left(a_{31}+b_{31}\right)\left(a_{31}+b_{31}\right)>0, \quad \lim f(y)=\infty$, and $f^{\prime}(y)=3 y^{2}+2 M_{9} y+M_{10}$. Hence, ${ }^{y} \overrightarrow{\mathrm{we}}$ assume that $\left(H_{7}\right): \quad \Delta_{4}=M_{9}^{2}-3 M_{10} \leq 0 ; \quad\left(H_{8}\right): \quad \Delta_{4}=M_{9}^{2}-3 M_{10}>0$, $y_{+}=\left(\left(-M_{9}+\sqrt{\Delta_{4}}\right) / 3\right)>0$, and $f\left(y_{+}\right) \leq 0$.

From Lemmas 2.2 and 4.2 in [50], we obtain the following lemma.

Lemma 1. For polynomial equation (30), the following conclusions are true.

(1) If the assumption $\left(H_{7}\right)$ holds, equation (30) has no positive root

(2) If the assumption $\left(H_{8}\right)$ holds, equation (30) has at least one positive root

Without losing of generality, we assume that equation (30) has three positive roots, denoted by $y_{1}, y_{2}$, and $y_{3}$. Hence, equation (29) has three positive roots $\omega_{1}^{k}=\sqrt{y_{k}}(k=1,2$, and 3$)$. By equation (28), we can get that the critical values of time delay $\tau$ are

$$
\tau_{k}^{j}=\frac{1}{\omega_{1}^{k}} \arccos \left[\frac{F_{1}\left(\omega_{1}^{k}\right)^{4}+\left(F_{0} D_{2}-F_{1} D_{1}\right)\left(\omega_{1}^{k}\right)^{2}-F_{0} D_{0}}{F_{1}^{2}\left(\omega_{1}^{k}\right)^{2}+F_{0}^{2}}\right]+\frac{2 j \pi}{\omega_{1}^{k}},
$$

for $k=1,2$, and $3, j \in N_{0}$. Therefore, $\pm i \omega_{1}^{k}$ is a pair of purely imaginary roots of equation (25) when $\tau=\tau_{k}^{j}(k=1,2$, and $3 ; j=1,2, \ldots)$. $\tau_{0}=\tau_{0}^{0}=\min _{k=1,2,3, j \in N_{0}}\left\{\tau_{k}^{j}\right\}$ and $\omega_{1}^{0}=\left.\omega_{1}^{k}\right|_{\tau=\tau_{0}}$.

Denote

Let $\lambda(\tau)=\alpha(\tau) \pm \nu(\tau)$ be the root of equation (25) near $\tau=\tau_{k}^{j}$ with $\alpha\left(\tau_{k}^{j}\right)=0$ and $\nu\left(\tau_{k}^{j}\right)=\omega_{1}^{k}, k=1,2$, and 3 and $j \in N_{0}$. Then, we have the following transversality condition.

Lemma 2. Suppose that $f^{\prime}\left(y_{k}\right) \neq 0$; then, the following transversality condition is satisfied: $\operatorname{sign}\{(d \operatorname{Re}(\lambda(\tau)))$ / $d \tau\}_{\lambda=i \omega_{1}^{0}} \neq 0$.

Proof. Differentiating the two sides of equation (25) with respect to $\tau$, we have

$$
\left[\frac{\mathrm{d} \lambda}{\mathrm{d} \tau}\right]^{-1}=\frac{\left(3 \lambda^{2}+2 D_{2} \lambda+D_{1}\right) e^{\lambda \tau}+F_{1}}{\lambda\left(F_{1} \lambda+F_{0}\right)}-\frac{\tau}{\lambda}
$$

Then, we obtain

$$
\left\{\frac{\mathrm{d} \operatorname{Re}(\lambda(\tau))}{\mathrm{d} \tau}\right\}_{\lambda=i \omega_{1}^{0}}^{-1}=\operatorname{Re}\left\{\frac{\left(3 \lambda^{2}+2 D_{2} \lambda+D_{1}\right) e^{\lambda \tau}}{\lambda\left(F_{1} \lambda+F_{0}\right)}+\frac{F_{1}}{\lambda\left(F_{1} \lambda+F_{0}\right)}\right\}_{\lambda=i \omega_{1}^{0}} .
$$

By some calculation, we get

$$
\begin{aligned}
\left\{\frac{\mathrm{dRe}(\lambda(\tau))}{\mathrm{d} \tau}\right\}_{\lambda=i \omega_{1}^{0}}^{-1} & =\frac{3\left(\omega_{1}^{0}\right)^{6}+2\left(D_{2}^{2}-2 D_{1}\right)\left(\omega_{1}^{0}\right)^{4}+\left(D_{1}^{2}-2 D_{0} D_{2}-F_{1}^{2}\right)\left(\omega_{1}^{0}\right)^{2}}{\Lambda_{1}} \\
& =\frac{y_{k}}{\Lambda_{1}}\left[3 y_{k}^{2}+2\left(D_{2}^{2}-2 D_{1}\right) y_{k}+\left(D_{1}^{2}-2 D_{0} D_{2}-F_{1}^{2}\right)\right] \\
& =\frac{y_{k}}{\Lambda_{1}} f^{\prime}\left(y_{k}\right) \neq 0
\end{aligned}
$$

where $\Lambda_{1}=F_{1}^{2} \omega_{1}^{2}+F_{0}^{2}$.

Hence,

$$
\operatorname{sign}\left\{\frac{\mathrm{dRe}(\lambda(\tau))}{\mathrm{d} \tau}\right\}_{\lambda=i \omega_{1}^{0}}=\operatorname{sign}\left\{\frac{\mathrm{dRe}(\lambda(\tau))}{\mathrm{d} \tau}\right\}_{\lambda=i \omega_{1}^{0}}^{-1} \neq 0
$$

By the theory of Hopf bifurcation, we have the following result.
Theorem 6. Assume that $\left(H_{5}\right)$ and $\left(H_{6}\right)$ hold, then the following statements are true.

(1) If the hypothesis $\left(H_{7}\right)$ holds, then the coexistence equilibrium $E^{*}$ of model (21) is locally asymptotically stable for $\tau \geq 0$.

(2) If the hypothesis $\left(H_{8}\right)$ and $f^{\prime}\left(y_{k}\right) \neq$ $0(k=1,2$, and 3$)$ hold, then the coexistence equilibrium $E^{*}$ of model (21) is locally asymptotically 
stable for $\tau \in\left[0, \tau_{0}\right)$ and unstable for $\tau>\tau_{0}$. Moreover, model (21) undergoes Hopf bifurcation at the coexistence equilibrium $E^{*}$ when $\tau=\tau_{0}$.

3.2. The Properties of Hopf Bifurcation. In the previous parts, we have discussed the existence of Hopf bifurcation of model (21) at the coexistence equilibrium $E^{*}$. Next, we will study the direction of Hopf bifurcation and the stability of bifurcated periodic solution by using the normal form theory and center manifold theory developed by Hassard et al. [51].
Let $\mu=\tau-\tau_{0}$ with $\mu \in R$; then, $\mu=0$ is a Hopf bifurcation value of model (21). Let $u_{1}(t)=P(t)-P^{*}$, $u_{2}(t)=Z_{1}(t)-Z_{1}^{*}$, and $u_{3}(t)=Z_{2}(t)-Z_{2}^{*}$; then, model (21) can be rewritten in the phase space $\mathscr{C}=C\left(\left[-\tau_{0}, 0\right], R^{3}\right)$ :

$$
\dot{U}(t)=L U_{t}+F\left(\mu, U_{t}\right),
$$

where $U(t)=\left(u_{1}(t), u_{2}(t), u_{3}(t)\right)^{T} \in R^{3}, L: \mathscr{C} \longrightarrow R^{3}, F$ : $R \times \mathscr{C} \longrightarrow R^{3}, \varphi(\theta)=\left(\varphi_{1}(\theta), \varphi_{2}(\theta), \varphi_{3}(\theta)\right) \in R^{3}$ has the following:

$$
\begin{aligned}
L \varphi & =L_{1} \varphi(0)+L_{2} \varphi\left(-\tau_{0}-\mu\right), \\
F\left(\mu, U_{t}\right) & =\left(\begin{array}{c}
a_{14} \varphi_{1}^{2}(0)+a_{15} \varphi_{2}^{2}(0)+a_{16} \varphi_{1}(0) \varphi_{2}(0)+a_{17} \varphi_{1}(0) \varphi_{3}(0) \\
a_{24} \varphi_{1}^{2}(0)+a_{25} \varphi_{2}^{2}(0)+a_{16} \varphi_{1}(0) \varphi_{2}(0)+b_{24} \varphi_{1}^{2}\left(-\tau_{0}-\mu\right)+b_{25} \varphi_{1}\left(-\tau_{0}-\mu\right) \varphi_{2}(0) \\
a_{34} \varphi_{1}^{2}(0)+a_{35} \varphi_{1}(0) \varphi_{3}(0)+b_{34} \varphi_{1}^{2}\left(-\tau_{0}-\mu\right)+b_{35} \varphi_{1}\left(-\tau_{0}-\mu\right) \varphi_{3}(0)
\end{array}\right),
\end{aligned}
$$

where

$$
\begin{aligned}
& a_{14}=-\frac{1}{K}+\frac{\mu_{1} Z_{1}^{*}\left(\alpha_{1}+s Z_{1}^{*}\right)}{\left(\alpha_{1}+P^{*}+s Z_{1}^{*}\right)^{3}}+\frac{\mu_{2} \alpha_{2} Z_{2}^{*}}{\left(\alpha_{2}+P^{*}\right)^{3}}, \\
& a_{15}=\frac{\mu_{1} s P^{*}\left(\alpha_{1}+P^{*}\right)}{\left(\alpha_{1}+P^{*}+s Z_{1}^{*}\right)^{3}} \\
& a_{17}=-\frac{\mu_{2} \alpha_{2}}{\left(\alpha_{2}+P^{*}\right)^{2}}, \\
& a_{16}=-\frac{\mu_{1} \alpha_{1}\left(\alpha_{1}+P^{*}+s Z_{1}^{*}\right)+2 \mu_{1} s P^{*} Z_{1}^{*}}{\left(\alpha_{1}+P^{*}+s Z_{1}^{*}\right)^{3}} \\
& a_{24}=-\frac{\beta_{1} Z_{1}^{*}\left(\alpha_{1}+s Z_{1}^{*}\right)}{\left(\alpha_{1}+P^{*}+s Z_{1}^{*}\right)^{3}} \\
& a_{25}=\frac{\left(\beta_{1}-\rho_{1}\right) P^{*}\left(\alpha_{1}+P^{*}\right)}{\left(\alpha_{1}+P^{*}+s Z_{1}^{*}\right)^{3}}, \\
& a_{26}=\frac{\beta_{1} \alpha_{1}\left(\alpha_{1}+P^{*}+s Z_{1}^{*}\right)+2 \beta_{1} s P^{*} Z_{1}^{*}}{\left(\alpha_{1}+P^{*}+s Z_{1}^{*}\right)^{3}} \\
& a_{34}=-\frac{\beta_{2} \alpha_{2} Z_{2}^{*}}{\left(\alpha_{2}+P^{*}\right)^{2}}, \\
& a_{35}=\frac{\beta_{2} \alpha_{2}}{\left(\alpha_{2}+P^{*}\right)^{2}}, \\
& b_{25}=-\frac{\rho_{1} \alpha_{1}\left(\alpha_{1}+P^{*}+s Z_{1}^{*}\right)+2 \rho_{1} s P^{*} Z_{1}^{*}}{\left(\alpha_{1}+P^{*}+s Z_{1}^{*}\right)^{3}}, \\
& b_{24}=\frac{\rho_{1} Z_{1}^{*}\left(\alpha_{1}+s Z_{1}^{*}\right)}{\left(\alpha_{1}+P^{*}+s Z_{1}^{*}\right)^{3}} \\
& b_{35}=-\frac{\rho_{2} \alpha_{2}}{\left(\alpha_{2}+P^{*}\right)^{2}}, \\
& \left.\alpha_{2}+P^{*}\right)^{2}
\end{aligned}
$$

and $L_{1}$ and $L_{2}$ are given in (23).
According to the Riesz representation theorem [52], there exists a function whose elements are $\eta(\theta, \mu)\left(-\tau_{0}<\theta<0\right)$ of bounded variance such that

$$
L(\varphi)=\int_{-\tau_{0}}^{0} \mathrm{~d} \eta(\theta, \mu) \varphi(\theta), \quad \varphi \in C\left(\left[-\tau_{0}, 0\right], R^{3}\right) .
$$

Thus, we chose

$$
\eta(\theta, \mu)= \begin{cases}L_{1}, & \theta=0, \\ 0, & \theta \in\left(-\tau_{0}, 0\right), \\ -L_{2}, & \theta=-\tau_{0} .\end{cases}
$$

For $\varphi \in C^{1}\left(\left[-\tau_{0}, R^{3}\right]\right)$, we define the operator:

$$
\begin{aligned}
& A(\mu) \varphi(\theta)= \begin{cases}\frac{\mathrm{d} \varphi(\theta)}{\mathrm{d} \theta}, & \theta \in\left[-\tau_{0}, 0\right), \\
\int_{-\tau_{0}}^{0} \mathrm{~d} \eta(\theta, \mu) \varphi(\theta), & \theta=0,\end{cases} \\
& R(\mu) \varphi(\theta)= \begin{cases}0, & \theta \in\left[-\tau_{0}, 0\right), \\
F(\varphi, \mu), & \theta=0 .\end{cases}
\end{aligned}
$$

Then, system (36) is equivalent to

$$
\dot{U}(t)=A(\mu) U_{t}+R(\mu) U_{t} .
$$

For $\varphi \in C^{1}\left(\left[-\tau_{0}, 0\right], R^{3}\right), A^{*}$ is defined as the adjoint operator of $A$ as

$$
A_{*}(\psi(\xi))= \begin{cases}-\frac{\mathrm{d} \psi(\xi)}{\mathrm{d} \xi}, & \xi \in\left(0, \tau_{0}\right], \\ \int_{-\tau_{0}}^{0} \mathrm{~d} \eta^{T}(\zeta, \mu) \psi(-\zeta), & \xi=0 .\end{cases}
$$

The bilinear product is given by 


$$
\langle\psi, \varphi\rangle=\bar{\psi}(0) \varphi(0)-\int_{-\tau_{0}}^{0} \int_{0}^{\theta} \bar{\psi}(\xi-\theta) \mathrm{d} \eta(\theta) \varphi(\xi) \mathrm{d} \xi
$$

where $\eta(\theta)=\eta(\theta, 0)$.

By the discussion in Section 3.1, $\pm i \omega_{1}^{0}$ are the eigenvalues of $A(0)$ and $A^{*}(0)$, which satisfy $A(0) q(\theta)=i \omega_{1}^{0} q(\theta)$ and $A^{*}(0)=-i \omega_{1}^{0} q^{*}(\xi)$. Suppose that $q(\theta)=\left(1, q_{2}, q_{3}\right)^{T} e^{i \omega_{1}^{0} \theta}$ is the eigenvector of $A(0)$ corresponding to the eigenvalue $i \omega_{1}^{0}$ and $q^{*}(\xi)=D\left(1, q_{2}^{*}, q_{3}^{*}\right) e^{-i \omega_{1}^{0} \xi}$ is the eigenvector of $A^{*}(0)$ corresponding to the eigenvalue $-i \omega_{1}^{0}$. By computation, we have

$$
\begin{aligned}
& q_{1}=\frac{a_{21}}{i \omega_{1}^{0}-a_{22}}, \\
& q_{2}=\frac{\left(i \omega_{1}^{0}-a_{11}\right)\left(i \omega_{1}^{0}-a_{22}\right)-a_{12} a_{21}}{a_{13}\left(i \omega_{1}^{0}-a_{22}\right)}, \\
& q_{1}^{*}=-\frac{a_{12}}{i \omega_{1}^{0}+a_{22}}, \\
& q_{2}^{*}=-\frac{\left(i \omega_{1}^{0}+a_{11}\right)\left(i \omega_{1}^{0}+a_{22}\right)-a_{12}\left(a_{21}+b_{21} e^{i \omega_{1}^{0} \tau_{0}}\right)}{\left(a_{31}+b_{31} e^{i \omega_{1}^{0} \tau_{0}}\right)\left(i \omega_{1}^{0}+a_{22}\right)} .
\end{aligned}
$$

From equation (44) and $\left\langle q^{*}, q\right\rangle=1$, we can get

$$
\begin{aligned}
\left\langle q^{*}, q\right\rangle & =\bar{q}^{*}(0) q(0)-\int_{-\tau_{0}}^{0} \int_{0}^{\theta} \bar{q}^{*}(\xi-\theta) \mathrm{d} \eta(\theta) q(\xi) \mathrm{d} \xi \\
& =\bar{D}\left[\left(1, \bar{q}_{1}^{*}, \bar{q}_{2}^{*}\right)\left(1, q_{1}, q_{2}\right)^{T}-\int_{-\tau_{0}}^{0} \int_{0}^{\theta}\left(1, \bar{q}_{1}^{*}, \bar{q}_{2}^{*}\right)\left(1, q_{1}, q_{2}\right)^{T} e^{i \omega_{1}^{0} \theta} \mathrm{d} \eta(\theta) \mathrm{d} \xi\right] \\
& =\bar{D}\left[1+\bar{q}_{1}^{*} q_{1}+\bar{q}_{2}^{*} q_{2}+\tau_{0} e^{-i \omega_{1}^{0} \tau_{0}}\left(b_{21} \bar{q}_{1}^{*}+b_{31} \bar{q}_{2}^{*}\right)\right] \\
& =1
\end{aligned}
$$

Thus,

$$
\bar{D}=\left[1+\bar{q}_{1}^{*} q_{1}+\bar{q}_{2}^{*} q_{2}+\tau_{0} e^{-i \omega_{1}^{0} \tau_{0}}\left(b_{21} \bar{q}_{1}^{*}+b_{31} \bar{q}_{2}^{*}\right)\right]^{-1} .
$$

In the remainder of this section, through using the method in [51], we can get the following relevant parameters which can determine the direction of Hopf bifurcation and the stability of the bifurcation periodic solutions:

$$
\begin{aligned}
& g_{20}=2 \bar{D}\left(k_{11}+\bar{q}_{1}^{*} k_{21}+\bar{q}_{2}^{*} k_{31}\right), \\
& g_{11}=\bar{D}\left(k_{12}+\bar{q}_{1}^{*} k_{22}+\bar{q}_{2}^{*} k_{32}\right), \\
& g_{02}=2 \bar{D}\left(k_{13}+\bar{q}_{1}^{*} k_{23}+\bar{q}_{2}^{*} k_{33}\right), \\
& g_{21}=2 \bar{D}\left(k_{14}+\bar{q}_{1}^{*} k_{24}+\bar{q}_{2}^{*} k_{34}\right),
\end{aligned}
$$


where

$$
\begin{aligned}
& k_{11}=a_{14}+q_{1}^{2} a_{15}+q_{1} a_{16}+q_{2} a_{17} \\
& k_{13}=a_{14}+\bar{q}_{1}^{2} a_{15}+\bar{q}_{1} a_{16}+\bar{q}_{2} a_{17} \\
& k_{12}=2 a_{14}+2 q_{1} \bar{q}_{1} a_{15}+\left(q_{1}+\bar{q}_{1}\right) a_{16}+\left(q_{2}+\bar{q}_{2}\right) a_{17}, \\
& k_{21}=a_{24}+q_{1}^{2} a_{25}+q_{1} a_{26}+e^{-2 i \omega_{1}^{0} \tau_{0}} b_{24}+q_{1} e^{i \omega_{1}^{0} \tau_{0}} b_{25}, \\
& k_{22}=2 a_{24}+2 q_{1} \bar{q}_{1} a_{25}+\left(q_{1}+\bar{q}_{1}\right) a_{26}+2 b_{24}+\left(q_{1} e^{i \omega_{1}^{0} \tau_{0}}+\bar{q}_{1} e^{-i \omega_{1}^{0} \tau_{0}}\right) b_{25}, \\
& k_{23}=a_{24}+\bar{q}_{1}^{2} a_{25}+\bar{q}_{1} a_{26}+e^{2 i \omega_{1}^{0} \tau_{0}} b_{24}+\bar{q}_{1} e^{-i \omega_{1}^{0} \tau_{0}} b_{25}, \\
& k_{31}=a_{34}+q_{2} a_{35}+e^{-2 i \omega_{1}^{0} \tau_{0}} b_{34}+q_{2} e^{-i \omega_{1}^{0} \tau_{0}} b_{35}, \\
& k_{32}=a_{34}+\left(q_{2}+\bar{q}_{2}\right) a_{35}+2 b_{34}+\left(q_{2} e^{i \omega_{1}^{0} \tau_{0}}+\bar{q}_{1} e^{-i \omega_{1}^{0} \tau_{0}}\right) b_{35}, \\
& k_{33}=a_{34}+\bar{q}_{2} a_{35}+e^{2 i \omega_{1}^{0} \tau_{0}} b_{34}+\bar{q}_{2} e^{i \omega_{1}^{0} \tau_{0}} b_{35} \\
& k_{14}=a_{14}\left(W_{20}^{(1)}(0)+2 W_{11}^{(1)}(0)\right)+a_{15}\left(\bar{q}_{1} W_{20}^{(2)}(0)+2 q_{1} W_{11}^{(2)}(0)\right)+a_{16}\left(\frac{1}{2} W_{20}^{(2)}(0)+W_{11}^{(2)}(0)+q_{1} W_{11}^{(1)(0)}+\frac{1}{2} \bar{q}_{1} W_{20}^{(1)}(0)\right) \\
& +a_{17}\left(\frac{1}{2} W_{20}^{(3)}(0)+\frac{1}{2} \bar{q}_{2} W_{20}^{(1)}(0)+W_{11}^{(3)}(0)+q_{2} W_{11}^{(1)}(0)\right) \\
& k_{24}=a_{24}\left(W_{20}^{(1)}(0)+2 W_{11}^{(1)}(0)\right)+a_{25}\left(\bar{q}_{1} W_{20}^{(2)}(0)+2 q_{1} W_{11}^{(2)}(0)\right)+a_{26}\left(\frac{1}{2} W_{20}^{(2)}(0)+W_{11}^{(2)}(0)+q_{1} W_{11}^{(1)}(0)+\frac{1}{2} \bar{q}_{1} W_{20}^{(1)}(0)\right) \\
& +b_{24}\left(e^{i \omega_{1}^{0} \tau_{0}} W_{20}^{(1)}\left(-\tau_{0}\right)+2 e^{-i \omega_{1}^{0} \tau_{0}} W_{11}^{(2)}\left(-\tau_{0}\right)\right)+b_{25}\left(\frac{1}{2} \bar{q}_{1} W_{20}^{(1)}\left(-\tau_{0}\right)+q_{1} W_{11}^{(1)}\left(-\tau_{0}\right)+e^{-i \omega_{1}^{0} \tau_{0}} W_{11}^{(2)}(0)+\frac{1}{2} e^{i \omega_{1}^{0} \tau_{0}} W_{20}^{(2)}\right) \\
& k_{34}=a_{34}\left(W_{20}^{(1)}(0)+2 W_{11}^{(1)}(0)\right)+a_{35}\left(\frac{1}{2} W_{20}^{(3)}(0)+\frac{1}{2} \bar{q}_{2} W_{20}^{(1)}(0)+W_{11}^{(3)}(0)+q_{2} W_{11}^{(1)}(0)\right) \\
& +b_{34}\left(e^{i \omega_{1}^{0} \tau_{0}} W_{20}^{(1)}\left(-\tau_{0}\right)+2 e^{-i \omega_{1}^{0} \tau_{0}} W_{11}^{(2)}\left(-\tau_{0}\right)\right) \\
& +b_{35}\left(q_{2} W_{11}^{(1)}\left(-\tau_{0}\right)+\frac{1}{2} \bar{q}_{2} W_{20}^{(1)}\left(-\tau_{0}\right)+\frac{1}{2} e^{i \omega_{1}^{0} \tau_{0}} W_{20}^{(3)}(0)+e^{-i \omega_{1}^{0} \tau_{0}} W_{11}^{(3)}(0)\right) \\
& W_{20}(\theta)=\frac{i g_{20} q(0)}{\omega_{1}^{0}} e^{i \omega_{1}^{0} \theta}+\frac{i \bar{g}_{02} \bar{q}(0)}{3 \omega_{1}^{0}} e^{-i \omega_{1}^{0} \theta}+E_{1} e^{2 i \omega_{1}^{0} \theta} \\
& W_{11}(\theta)=-\frac{i g_{11} q(0)}{\omega_{1}^{0}} e^{i \omega_{1}^{0} \theta}+\frac{i \bar{g}_{11} \bar{q}(0)}{\omega_{1}^{0}} e^{-i \omega_{1}^{0} \theta}+E_{2} \text {, }
\end{aligned}
$$

where $E_{1}=\left(E_{1}^{(1)}, E_{1}^{(2)}, E_{1}^{(3)}\right)^{T} \in R^{3}$ and $E_{2}=\left(E_{2}^{(1)}, E_{2}\right.$ $\left.(2), E_{2}^{(3)}\right)^{T} \in R^{3}$ can be determined by the following, respectively: 


$$
\begin{aligned}
& E_{1}=2\left(\begin{array}{ccc}
2 i \omega_{1}^{0}-a_{11} & -a_{12} & -a_{13} \\
-a_{21}-b_{21} e^{-2 i \omega_{1}^{0} \tau_{0}} & 2 i \omega_{1}^{0}-a_{22} & 0 \\
-a_{31}-b_{31} e^{-2 i \omega_{1}^{0} \tau_{0}} & 0 & 2 i \omega_{1}^{0}
\end{array}\right)^{-1}\left(\begin{array}{l}
k_{11} \\
k_{21} \\
k_{31}
\end{array}\right), \\
& E_{2}=\left(\begin{array}{ccc}
-a_{11} & -a_{12} & -a_{13} \\
-a_{21}-b_{21} & -a_{22} & 0 \\
-a_{31}-b_{31} & 0 & 0
\end{array}\right)^{-1}\left(\begin{array}{l}
k_{12} \\
k_{22} \\
k_{32}
\end{array}\right) .
\end{aligned}
$$

So we can get $g_{21}$ and compute the following values:

$$
\begin{aligned}
c_{1}(0) & =\frac{i}{2 \omega_{1}^{0} \tau_{0}}\left(g_{20} g_{11}-2\left|g_{11}\right|^{2}-\frac{\left|g_{02}\right|^{2}}{3}\right)+\frac{g_{21}}{2}, \\
v_{1} & =-\frac{\operatorname{Re}\left\{c_{1}(0)\right\}}{\operatorname{Re}\left\{\lambda^{\prime}\left(\tau_{0}\right)\right\}}, \\
\varrho_{1} & =2 \operatorname{Re}\left\{c_{1}(0)\right\}, \\
T_{1} & =-\frac{\operatorname{Im}\left\{c_{1}(0)\right\}+v_{1} \operatorname{Im}\left\{\lambda^{\prime}\left(\tau_{0}\right)\right\}}{\omega_{1}^{0} \tau_{0}},
\end{aligned}
$$

which determine the direction of Hopf bifurcation and the stability of bifurcating periodic solutions when $\tau=\tau_{0}$. From the conclusions of Hassard et al. [51], we have the following results.

Theorem 7. Suppose $\left(H_{5}\right),\left(H_{6}\right),\left(H_{8}\right)$, and $f^{\prime}\left(y_{i}\right)(i=$ $1,2$, and 3$)$ are satisfied. For the properties of Hopf bifurcation of model (21), we have that

(1) If $v_{1}>0(<0)$, then the direction of the Hopf bifurcation is forward (backward) and the bifurcating periodic solutions exist for $\tau>\tau_{0}\left(\tau<\tau_{0}\right)$

(2) If $\varrho_{1}<0(>0)$, then the bifurcating periodic solutions are orbitally stable (unstable)

(3) If $T_{1}>0(<0)$, then the period of bifurcation periodic solutions increase (decrease)

\section{Hopf Bifurcation of System (3)}

4.1. The Existence of Hopf Bifurcation of System (3). In this section, we study the dynamic behavior of system (3) at the coexistence equilibrium. According to the properties of the Laplacian operator defined on the bounded domain with homogeneous Neumann boundary conditions [53], we get that the operator $\Delta$ has the eigenvalues $-\left(n^{2} / l^{2}\right)\left(\left(n \in N_{0} \triangleq\{0,1,2, \ldots\}\right)\right.$ with the corresponding eigenfunctions on $X$ denoted by $\eta_{n}^{1}=(\cos n x, 0,0)^{T}, \eta_{n}^{2}$ $=(0, \cos n x, 0)^{T}$, and $\eta_{n}^{3}=(0,0, \cos n x)^{T}\left(n \in N_{0}\right)$. Here, we assume that $l=1$.

Under the condition $\left(H_{4}\right)$, the linearization of system (3) at $E^{*}$ in the phase space $\mathscr{C}=C([-\tau, 0], X)$ can be rewritten as follows:

$$
\frac{\mathrm{d} U(t)}{\mathrm{d} t}=D \Delta U(t)+L_{1}(U(t))+L_{2}(U(t-\tau))
$$

where $D=\operatorname{diag}\left(d_{1}, d_{2}, d_{3}\right), L_{1}$ and $L_{2}$ are defined in Section 3.1, and $U(t)=\left(u_{1}(t), u_{2}(t), u_{3}(t)\right)^{T}=\left(u_{1}(t, \cdot), u_{2}(t, \cdot)\right.$, $\left.u_{3}(t, \cdot)\right)^{T}$.

The characteristic equation of model (52) is

$$
P(\lambda, \tau)=\lambda^{3}+h_{2 n} \lambda^{2}+h_{1 n} \lambda+h_{0 n}+e^{-\lambda \tau}\left(c_{1 n} \lambda+c_{0 n}\right)=0,
$$

where

$$
\begin{aligned}
& h_{2 n}=d_{1} n^{2}+d_{2} n^{2}+d_{3} n^{2}-a_{11}-a_{22}, c_{1 n}=-\left(a_{12} b_{21}+a_{13} b_{31}\right), \\
& h_{1 n}=\left(d_{1} d_{2}+d_{1} d_{3}+d_{2} d_{3}\right) n^{4}-\left(a_{11}+a_{22}\right)\left(d_{2}+d_{3}\right) n^{2}+a_{11} a_{22}-a_{12} a_{21}-a_{13} a_{31}, \\
& h_{0 n}=d_{1} d_{2} d_{3} n^{6}-d_{3}\left(d_{1} a_{22}+d_{2} a_{11}\right) n^{4}+d_{3} n^{2}\left(a_{11} a_{22}-a_{12} a_{21}\right)-a_{13} a_{31} d_{2} n^{2}+a_{13} a_{31} a_{22}, \\
& c_{0 n}=a_{13} a_{22} b_{31}-d_{3} n^{2} a_{12} b_{21}-d_{2} n^{2} a_{13} b_{31} .
\end{aligned}
$$

Next, we seek for condition determining the stability of the coexistence equilibrium $E^{*}$ of system (3) with $\tau=0$. For this purpose, substituting $\tau=0$ into equation (53), we obtain that

$$
P(\lambda, 0)=\lambda^{3}+h_{2 n} \lambda^{2}+\left(h_{1 n}+c_{1 n}\right) \lambda+h_{0 n}+c_{0 n}=0 .
$$

Simple computation yields that 


$$
\begin{aligned}
h_{1 n}+c_{1 n}= & \left.\left(d_{1} d_{2}+d_{1} d_{3}+d_{2} d_{3}\right) n^{4}-a_{11}\left(d_{2}+d_{3}\right) n^{2}-a_{22}\left(d_{1}+d_{3}\right)\right) n^{2} \\
& +a_{11} a_{22}-a_{12} a_{21}-a_{13} a_{31}-a_{12} b_{21}-a_{13} b_{31}, \\
h_{0 n}+c_{0 n}= & d_{1} d_{2} d_{3} n^{6}-d_{3}\left(d_{1} a_{22}+d_{2} a_{11}\right) n^{4}+d_{3} n^{2}\left(a_{11} a_{22}-a_{12} a_{21}-a_{12} b_{21}\right) \\
& -d_{2} n^{2}\left(a_{13} b_{31}+a_{13} a_{31}\right)+a_{13} a_{31} a_{22}+a_{13} a_{22} b_{31}, \\
h_{2 n}\left(h_{1 n}+c_{1 n}\right)-h_{0 n}-c_{0 n}= & d_{1 n} n^{2}\left(h_{1 n}+c_{1 n}-d_{2} d_{3} n^{4}\right)+d_{2 n} n^{2}\left(h_{1 n}+c_{1 n}+a_{13} b_{31}+a_{13} a_{31}\right) \\
& +d_{3} n^{2}\left(h_{1 n}+c_{1 n}-a_{11} a_{22}+a_{12} a_{21}+a_{12} b_{21}\right)-a_{11}\left(h_{1 n}+c_{1 n}\right)-a_{22}\left(h_{1 n}+c_{1 n}+a_{13} a_{31}+a_{13} b_{31}\right) .
\end{aligned}
$$

Under the condition $\left(H_{6}\right), h_{1 n}+c_{1 n}>0, h_{0 n}+c_{0 n}>0$, and $h_{2 n}\left(h_{1 n}+c_{1 n}\right)-h_{0 n}-c_{0 n}>0$, for all $n \in N_{0}$. Through the Routh-Hurwitz criterion [48], all the roots of equation (55) have negative real parts. From Theorems 5.1.1 and 5.1.3 of Henry [54], system (3) at $E^{*}$ is locally asymptotically stable when $\tau=0$. To sum up, diffusion does not change the stability of system (3) at equilibrium $E^{*}$. Then, we can get the following theorem.

Theorem 8. Assume the conditions $\left(\mathrm{H}_{5}\right)$ and $\left(\mathrm{H}_{6}\right)$ hold, then the coexistence equilibrium $E^{*}\left(\bar{P}_{1} \bar{Z}_{1}, \bar{Z}_{2}\right)$ of system (3) with $\tau=0$ is locally asymptotically stable.

Now, we study the effect of the delay $\tau \neq 0$ on the stability of the positive equilibrium $E^{*}$ of system (3). First, we seek critical values of $\tau$ such that equation (53) has a pair of purely imaginary roots.

Let $\lambda=i \omega_{2}\left(\omega_{2}>0\right)$ be a root of equation (53); then,

$$
-i \omega_{2}^{3}-h_{2 n} \omega_{2}^{2}+i h_{1 n} \omega_{2}+h_{0 n}+e^{-i \omega_{2} \tau}\left(i c_{1 n} \omega_{2}+c_{0 n}\right)=0,
$$

which we get

$$
\left\{\begin{array}{l}
-\omega_{2}^{3}+h_{1 n} \omega_{2}=c_{0 n} \sin \omega_{2} \tau-c_{1 n} \omega_{2} \cos \omega_{2} \tau, \\
-\omega_{2}^{2} h_{2 n}+h_{0 n}=-c_{1 n} \omega_{2} \sin \omega_{2} \tau-c_{0 n} \cos \omega_{2} \tau .
\end{array}\right.
$$

Two equations of equation (58) are squared on both sides and then added, which yields

$\omega_{2}^{6}+\left(h_{2 n}^{2}-2 h_{1 n}\right) \omega_{2}^{4}+\left(h_{1 n}^{2}-2 h_{0 n} h_{2 n}-c_{1 n}^{2}\right) \omega_{2}^{2}+h_{0 n}^{2}-c_{0 n}^{2}=0$.

Let $\omega_{2}^{2}=y_{1}$; then, equation (59) becomes

$$
g\left(y_{1}\right)=y_{1}^{3}+p_{n} y_{1}^{2}+q_{n} y_{1}+e_{n}=0,
$$

where

$$
\begin{aligned}
& p_{n}=h_{2 n}^{2}-2 h_{1 n}=\left(d_{1}^{2}+d_{2}^{2}+d_{3}^{2}\right) n^{4}-2 d_{1} n^{2} a_{11}-2 d_{2} n^{2} a_{22}+a_{11}^{2}+a_{22}^{2}+2 a_{12} a_{21}+2 a_{13} a_{31} \text {, } \\
& q_{n}=h_{1 n}^{2}-2 h_{0 n} h_{2 n}-c_{1 n}^{2}=\mathscr{F}(n)+\left(a_{11} a_{22}-a_{12} a_{21}-a_{13} a_{31}\right)^{2}-2\left(a_{11} a_{22}-a_{12} a_{21}-a_{13} a_{31}\right) a_{13} a_{31} a_{22}-\left(a_{12} b_{21}+a_{13} b_{31}\right)^{2} \text {, } \\
& e_{n}=h_{0 n}^{2}-c_{0 n}^{2} \text {, } \\
& h_{0 n}-c_{0 n}=d_{1} d_{2} d_{3} n^{6}-d_{3}\left(d_{1} a_{22}+d_{2} a_{11}\right) n^{4}+d_{3}\left(a_{11} a_{22}-a_{12} a_{21}+a_{12} b_{21}\right) n^{2}+d_{2} a_{13}\left(b_{31}-a_{31}\right) n^{2}+a_{22} a_{13}\left(a_{31}-b_{31}\right) \text {, } \\
& \mathscr{F}(n)=d_{1} d_{2} d_{3}\left(d_{1} d_{2}+d_{1} d_{3}+d_{2} d_{3}\right) n^{10}+\left[\left(d_{1} d_{2}+d_{1} d_{3}+d_{2} d_{3}\right)^{2}+d_{3}\left(d_{1} d_{2}+d_{1} d_{3}+d_{2} d_{3}\right)\right. \\
& \left.\left(d_{1} a_{22}+d_{2} a_{11}\right)+2 a_{11}\left(d_{2}+d_{3}\right) d_{1} d_{2} d_{3}+2 a_{22}\left(d_{1}+d_{3}\right) d_{1} d_{2} d_{3}\right] n^{8}+2\left[-a_{11}\left(d_{2}+d_{3}\right)\left(d_{1} d_{2}+d_{1} d_{3}+d_{2} d_{3}\right)\right. \\
& -a_{22}\left(d_{1}+d_{3}\right)\left(d_{1} d_{2}+d_{1} d_{3}+d_{2} d_{3}\right) \\
& -\left(d_{1} d_{2}+d_{1} d_{3}+d_{2} d_{3}\right) d_{3}\left(a_{11} a_{22}-a_{12} a_{21}\right)+a_{13} a_{31} d_{2}\left(d_{1} d_{2}+d_{1} d_{3}+d_{2} d_{3}\right)-a_{11} d_{3}\left(d_{2}+d_{3}\right)\left(d_{1} a_{22}+d_{2} a_{11}\right)-a_{22}\left(d_{1}+d_{3}\right) \\
& \left.\left(d_{1} a_{22}+d_{2} a_{11}\right)-\left(a_{11} a_{22}-a_{12} a_{21}-a_{13} a_{31}\right) d_{1} d_{2} d_{3}\right] n^{6}+\left[a_{11}^{2}\left(d_{2}+d_{3}\right)^{2}+a_{22}^{2}\left(d_{2}+d_{3}\right)^{2}\right. \\
& +2\left(a_{11} a_{22}-a_{12} a_{21}-a_{13} a_{31}\right)\left(d_{1} d_{2}+d_{1} d_{3}+d_{2} d_{3}\right)+2 a_{11} a_{22}\left(d_{2}+d_{3}\right)\left(d_{2}+d_{3}\right)-2 a_{13} a_{31} a_{22}\left(d_{1} d_{2}+d_{1} d_{3}+d_{2} d_{3}\right) \\
& +2 a_{11} d_{3}\left(d_{2}+d_{3}\right)\left(d_{1} a_{22}+d_{2} a_{11}\right)-2 a_{11} a_{13} a_{31} d_{2}\left(d_{2}+d_{3}\right)+2 a_{22} d_{3}\left(d_{1}+d_{3}\right)\left(a_{11} a_{22}+a_{12} a_{21}\right) \\
& \left.-2 a_{22} a_{13} a_{31} d_{2}\left(d_{1}+d_{3}\right)+2\left(a_{11} a_{22}-a_{12} a_{21}-a_{13} a_{31}\right) d_{3}\left(d_{1} a_{22}+d_{2} a_{11}\right)\right] n^{4}+\left[-2 a_{11} d_{3}\left(d_{2}+d_{3}\right)\left(a_{11} a_{22}-a_{12} a_{21}+a_{13} a_{31}\right)\right. \\
& -2 a_{22}\left(d_{1}+d_{3}\left(a_{11} a_{22}-a_{12} a_{21}+a_{13} a_{31}\right)+2 a_{11} a_{22} a_{13} a_{31}\left(d_{2}+d_{3}\right)+2 a_{22}^{2}\right) \\
& \left.a_{13} a_{31}\left(d_{1}+d_{2}\right)-2\left(a_{11} a_{22}-a_{12} a_{21}+a_{13} a_{31}\right) d_{3}\left(a_{11} a_{22}-a_{12} a_{21}\right)+2\left(a_{11} a_{22}-a_{12} a_{21}+a_{13} a_{31}\right) a_{13} a_{31} d_{2}\right] n^{2} .
\end{aligned}
$$


Under the condition $\left(H_{6}\right)$, for all $n \in N_{0}, g(0)=e_{n}$ $=h_{0 n}^{2}-c_{0 n}^{2}>0, \lim _{y_{1} \longrightarrow \infty} g\left(y_{1}\right)=\infty$, and $g^{\prime}\left(y_{1}\right)=3 y_{1}^{2}+2 p_{n} y_{1}$ $+q_{n}$.

From Lemmas 2.2 and 4.2 in [50], we obtain the following lemma.

Lemma 3. For polynomial equation (60), we can get the following conclusions about the distribution of its positive roots.

(1) If there exists $n_{1} \in N_{0}$, such that $p_{n_{1}}>0, q_{n_{1}}>0$, and $e_{n_{1}}>0$, then equation (60) has no positive roots

(2) If there exists $n_{2} \in N_{0}$, such that $e_{n_{2}}<0$, then equation (60) has at least one positive root

(3) If $e_{n} \geq 0$ and $\Delta_{5}=p_{n}^{2}-3 q_{n}>0$, then equation (60) has positive roots if and only if $y_{1_{+}}=\left(\left(-p_{n}+\sqrt{\Delta_{5}}\right) / 3\right)>0$ and $g\left(y_{1_{+}}\right) \leq 0$
Next, we assume that $\left(H_{9}\right): \quad \Delta_{6}=p_{0}^{2}-3 q_{0}>0$, $y_{1_{+}}=\left(\left(-p_{0}+\sqrt{\Delta_{6}}\right) / 3\right)>0$, and $g\left(y_{1_{+}}\right) \leq 0$. Thus, we have the following result.

Theorem 9. If the conditions $\left(H_{6}\right)$ and $\left(H_{9}\right)$ hold, then equation (60) has at least one positive root for $n=0$.

Proof. Under the condition $\left(H_{6}\right)$, then $h_{00}+c_{00}>0$ and $h_{00}-c_{00}>0$; hence, $e_{0}>0$. If $\left(H_{9}\right)$ holds, according to Lemma 3, then the proof is completed.

Assume that equation (60) has at least one positive root for $n=0$. Without loss of generality, we suppose that it has three positive roots, denoted by $y_{1_{1,0}}, y_{1_{2,0}}$, and $y_{1_{3,0}}$. Hence, equation (59) has three positive roots $\omega_{2_{k, 0}}=\sqrt{y_{1_{k, 0}}}$, $k=1,2$, and 3 . Through equation (58), we can get

$$
\tau_{k, j}^{0}=\frac{1}{\omega_{2_{k, 0}}}\left\{\arccos \left(\frac{c_{10} \omega_{2_{k, 0}}^{4}+\left(h_{20} c_{00}-h_{10} c_{10}\right) \omega_{2_{k, 0}}^{2}-h_{00} c_{00}}{c_{10}^{2} \omega_{2_{k, 0}}^{2}+c_{00}^{2}}\right)+2 j \pi\right\},
$$

for $k=1,2$, and $3, j \in N_{0}$. So $\pm i \omega_{2_{k, 0}}$ is a pair of purely imaginary roots of equation (55) with $\tau=\tau_{k, j}^{0}$. Define $\tau_{0}^{0}=\min _{k=1,2,3, j \in N_{0}}\left\{\tau_{k, j}^{0}\right\}$, and $\omega_{2}^{0}=\left.\omega_{2_{k, 0}}\right|_{\tau=\tau_{0}^{0}}$.

Let $\lambda(\tau)=\sigma(\tau) \pm \varepsilon(\tau) i$ be the roots of equation (53) near $\tau=\tau_{k, j}^{0}$; then, $\sigma\left(\tau_{k, j}^{0}\right)=0$ and $\varepsilon\left(\tau_{k, j}^{0}\right)=\omega_{2_{k, 0}}\left(j \in N_{0}\right)$. So we get the following transversality condition.
Proof. Differentiating the two sides of equation (53) with respect to $\tau$ yields

$$
\left[\frac{\mathrm{d} \lambda}{\mathrm{d} \tau}\right]^{-1}=\frac{3 \lambda^{2}+2 h_{20} \lambda+h_{10}}{\lambda\left(c_{10} \lambda+c_{00}\right)}+\frac{c_{10}}{\lambda\left(c_{10} \lambda+c_{00}\right)}-\frac{\tau}{\lambda} .
$$

Thus, we have

Lemma 4. Assume that $g^{\prime}\left(y_{1_{k, 0}}\right) \neq 0$; then, the following transversality condition holds: $\operatorname{sign}\{(d \operatorname{Re}(\lambda(\tau))) / d \tau\}_{\lambda=i \omega_{2}^{0}} \neq 0$.

$$
\left\{\frac{\operatorname{dRe}(\lambda(\tau))}{\mathrm{d} \tau}\right\}_{\lambda=i \omega_{2}^{0}}^{-1}=\operatorname{Re}\left\{\frac{3 \lambda^{2}+2 h_{20} \lambda+h_{10}}{\lambda\left(c_{10} \lambda+c_{00}\right)}\right\}_{\lambda=i \omega_{2}^{0}}+\operatorname{Re}\left\{\frac{c_{10}}{\lambda\left(c_{10} \lambda+c_{00}\right)}\right\}_{\lambda=i \omega_{2}^{0}}
$$

Via calculations, we get

$$
\begin{aligned}
\left\{\frac{\mathrm{dRe}(\lambda(\tau))}{\mathrm{d} \tau}\right\}_{\lambda=i \omega_{2}^{0}}^{-1} & =\frac{3\left(\omega_{2}^{0}\right)^{6}+2\left(h_{20}^{2}-2 h_{10}\right)\left(\omega_{2}^{0}\right)^{4}+\left(h_{10}^{2}-2 h_{20} h_{00}-c_{10}^{2}\right)\left(\omega_{2}^{0}\right)^{2}}{\Lambda_{2}} \\
& =\frac{y_{1_{k, 0}}}{\Lambda_{2}}\left[3 y_{1_{k, 0}}^{2}+2\left(h_{20}^{2}-2 h_{10}\right) y_{1_{k, 0}}+\left(h_{10}^{2}-2 h_{20} h_{00}-c_{10}^{2}\right)\right]=\frac{y_{1_{k, 0}}}{\Lambda_{2}} g^{\prime}\left(y_{1_{k, 0}}\right) \neq 0
\end{aligned}
$$

where $\Lambda_{2}=c_{10}\left(\omega_{2}^{0}\right)^{4}+c_{00}\left(\omega_{2}^{0}\right)^{2}$. Then, we have

$$
\operatorname{sign}\left\{\frac{\mathrm{dRe}(\lambda(\tau))}{\mathrm{d} \tau}\right\}_{\lambda=i \omega_{2}^{0}}=\operatorname{sign}\left\{\frac{\mathrm{dRe}(\lambda(\tau))}{\mathrm{d} \tau}\right\}_{\lambda=i \omega_{2}^{0}}^{-1} \neq 0
$$

This completes the proof of the lemma.
By the theory of Hopf bifurcation in $\mathrm{Wu}$ [55], we have the results on the existence of spatially homogeneous Hopf bifurcation.

Theorem 10. Suppose that the conditions $\left(H_{5}\right)$ and $\left(H_{6}\right)$ hold, then the following statements about system (3) are true. 
(i) If the condition $\left(H_{8}\right)$ holds, then the coexistence equilibrium $E^{*}$ of system (3) is locally asymptotically stable for $\tau \in\left[0, \tau_{0}^{0}\right) ; E^{*}$ is unstable when $\tau>\tau_{0}^{0}$

(ii) If $g^{\prime}\left(y_{1_{k, 0}}\right) \neq 0, k=1,2$, and 3 , holds, then system (3) undergoes a spatially homogeneous Hopf bifurcation at the coexistence equilibrium $E^{*}$ when $\tau=\tau_{0}^{0}$

To study the existence of spatially inhomogeneous Hopf bifurcation, we need to seek the conditions which satisfy that equation (60) has at least one positive root. From Lemma 3, we know that there exists $N_{1} \in N_{0}\left(N_{1} \geq 1\right)$, such that $p_{N_{1}}>0, q_{N_{1}}>0$, and $e_{N_{1}}>0$. When, $n>N_{1}$; equation (60) has no positive roots. When $n<N_{1}, \Delta_{5}=p_{n}^{2}-3 q_{n}>0$, $y_{1_{+}}=\left(\left(-p_{n}+\sqrt{\Delta_{5}}\right) / 3\right)>0$, and $g\left(y_{1_{+}}\right) \leq 0$ are satisfied. We know that equation (60) has at least one positive root for $1 \leq n \leq N_{1}$. Without loss of generality, we assume that equation (60) has three positive roots, which are denoted by $y_{1_{k, n_{0}}}, k=1,2$, and 3 . Then, equation (59) has three positive roots $\omega_{2_{k, n_{0}}}=\sqrt{y_{1_{k, n_{0}}}}$.

From equation (58), we can get

$$
\begin{aligned}
\tau_{k, j}^{n_{0}} & =\frac{1}{\omega_{2_{k, n_{0}}}}\left\{\frac{c_{1 n_{0}}\left(\omega_{2_{k, n_{0}}}\right)^{4}+\left(h_{2 n_{0}} c_{0 n_{0}}-c_{1 n_{0}} h_{1 n_{0}}\right)\left(\omega_{2_{k, n_{0}}}\right)^{2}+c_{0 n_{0}} h_{0 n_{0}}}{c_{0 n_{0}}+c_{1 n_{0}}^{2}\left(\omega_{2_{k, n_{0}}}\right)^{2}}\right\}+\frac{2 j \pi}{\omega_{2_{k, n_{0}}}}, \\
k & =1,2 \text {, and } 3 \text { and } j \in N_{0} .
\end{aligned}
$$

Then, $\pm i \omega_{2_{k, n_{0}}}$ is a pair of purely imaginary roots of equation (53) with $\tau=\tau_{k, j}^{n_{0}}$. Since $g^{\prime}\left(y_{1_{k, n_{0}}}\right) \neq 0$, using the same calculation method in Lemma 4 , we can get $\operatorname{sign}\{(\operatorname{dRe}(\lambda(\tau))) / \mathrm{d} \tau\}_{\tau=\tau_{k, j}^{n_{0}}} \neq 0$.

By the theory of Hopf bifurcation in Hassard et al. [51], we have the results on the existence of spatially inhomogeneous Hopf bifurcation.

Theorem 11. Suppose that the conditions $\left(H_{5}\right)$ and $\left(H_{6}\right)$ are true, and there exists $n_{0} \in N_{1}$ such that $p_{n_{0}}, q_{n_{n}}$, and $e_{n_{0}}$. Moreover, if $g^{\prime}\left(y_{k, n_{0}}\right) \neq 0(k=1,2$, and 3$)$ holds, then system (3) undergoes a spatially inhomogeneous Hopf bifurcation at $E^{*}$ when $\tau=\tau_{k, j}^{n_{0}}, k=1,2$, and 3 and $j \in N_{0}$.

To sum up, system (3) without diffusion undergoes a spatially homogeneous Hopf bifurcation at the coexistence equilibrium $E^{*}$. System (3) undergoes a spatially inhomogeneous Hopf bifurcation at the coexistence equilibrium $E^{*}$.

4.2. Direction and Stability of Bifurcated Periodic Solutions. We have get some conditions, such that a spatially homogeneous or inhomogeneous periodic solutions bifurcate from the coexistence equilibrium $E^{*}$ of system (3) when $\tau$ crosses through the critical values $\tau_{k, j}^{n}\left(k=1,2\right.$, and 3 and $\left.j \in N_{0}\right), n=0$ and $n_{0}$. In this part, we will study the direction of Hopf bifurcation and the stability of bifurcated periodic solutions by utilizing the normal form theory and center manifold theorem for partial functional differential equations [55]. For convenience, we fix $k=1,2$, and 3 and $j=0,1,2, \ldots$, and denote $\tau_{k, j}^{n}(n=0$ and $\left.n_{0}\right)$ by $\tilde{\tau}$ and $\omega_{2_{k, n}}\left(n=0\right.$ and $\left.n_{0}\right)$ by $\omega_{n}$.

First, if we introduce a new parameter $\tau=\widetilde{\tau}-v, v \in R$, then $v=0$ is the new Hopf bifurcation value. Let $\chi=(t / \tau)$ and rewrite $\chi$ as $t$; system (3) can be rewritten in the phase space $C_{1}=C_{1}([-1,0], X)$ as

$$
\dot{U}(t)=\tilde{\tau} D \Delta U(t)+\Gamma(\widetilde{\tau}) U(t)+H\left(U_{t}, v\right),
$$

where $u_{1}(t)=u_{1}(t, \cdot), u_{2}(t)=u_{2}(t, \cdot), u_{3}(t)=u_{3}(t, \cdot), U=$ $\left(u_{1}(t), u_{2}(t), u_{3}(t)\right)^{T}, D=\operatorname{diag}\left(d_{1}, d_{2}, d_{3}\right), \Gamma(\theta)(\cdot): C_{1} \longrightarrow$ $X$, and $H: C_{1} \times R \longrightarrow X$ are given by

$$
\begin{array}{r}
\Gamma(\theta) \varphi=\theta\left(\begin{array}{c}
a_{11} \varphi_{1}(0)+a_{12} \varphi_{2}(0)+a_{13} \varphi_{3}(0) \\
a_{21} \varphi_{1}(0)+a_{22} \varphi_{2}(0)+b_{21} \varphi_{1}(-1) \\
a_{31} \varphi_{1}(0)+b_{31} \varphi_{1}(-1)
\end{array}\right), \\
H(\varphi, v)=v D \Delta \varphi(0)+\Gamma(v) \varphi+h(\varphi, v),
\end{array}
$$

where

$$
h(\varphi, v)=(\tilde{\tau}+v)\left(\begin{array}{c}
a_{14} \varphi_{1}^{2}(0)+a_{15} \varphi_{2}^{2}(0)+a_{16} \varphi_{1}(0) \varphi_{2}(0)+a_{17} \varphi_{1}(0) \varphi_{3}(0) \\
a_{24} \varphi_{1}^{2}(0)+a_{25} \varphi_{2}^{2}(0)+a_{26} \varphi_{1}(0) \varphi_{2}(0)+b_{24} \varphi_{1}^{2}(-1) \\
+b_{25} \varphi_{1}(-1) \varphi_{2}(0) \\
a_{34} \varphi_{1}^{2}(0)+a_{35} \varphi_{1}(0) \varphi_{3}(0)+b_{34} \varphi_{1}^{2}(-1)+b_{35} \varphi_{1}(-1) \varphi_{3}(0)
\end{array}\right)
$$

for $\varphi=\left(\varphi_{1}, \varphi_{2}, \varphi_{3}\right)^{T} \in C_{1}$. 
Linearizing model (68) at the origin leads to the following equation:

$$
\dot{U}(t)=\tilde{\tau} D \Delta U(t)+\Gamma(\tilde{\tau}) U(t) .
$$

From the discussion in Section 4.1, we can get that the characteristic equation of equation (71) has a pair of purely imaginary roots $\Upsilon_{n}=\left\{i \omega_{n} \tilde{\tau},-i \omega_{n} \tilde{\tau}\right\}$ when $\tau=\tilde{\tau}$.

Let $\wp:=C_{1}\left([-1,0], R^{3}\right)$. Next, we consider the functional differential equation on $\wp$ :

$$
\dot{z}=\Gamma(\tilde{\tau})\left(z_{t}\right) .
$$

Obviously, $\Gamma(\tilde{\tau})$ is continuous linear function mapping $C_{1}\left([-1,0], R^{3}\right)$ into $R^{3}$. According to the Riesz representation theorem [52], there exists a $3 \times 3$ matrix function $\eta_{1}(\theta$, $\tilde{\tau}, n)(-1<\theta<0)$, whose entries are bounded variation that

$$
\Gamma(\widetilde{\tau})(\phi)=\int_{-1}^{0} \mathrm{~d} \eta_{1}(\theta, \widetilde{\tau}, n) \phi(\theta), \quad \phi \in C_{1}\left([-1,0], R^{3}\right) .
$$

We can choose

$$
\eta_{1}(\theta, \tilde{\tau}, n)=\tilde{\tau}\left(\begin{array}{ccc}
a_{11} & a_{12} & a_{13} \\
a_{21} & a_{22} & 0 \\
a_{31} & 0 & 0
\end{array}\right) \delta(\theta)-\tilde{\tau}\left(\begin{array}{ccc}
0 & 0 & 0 \\
b_{21} & 0 & 0 \\
b_{31} & 0 & 0
\end{array}\right) \delta(\theta+1)
$$

where $\delta$ is a Dirac delta function.

Define $C_{1}^{*}=C\left([-1,0], R^{3 *}\right)$, where $R^{3 *}$ is the three-dimensional vector space of row vectors. $A_{\tilde{\tau}}$ denotes the infinitesimal generator of semigroup induced by the solutions of equation (72), and $A_{\tau}^{*}$ is the adjoint matrix of $A_{\tau}$ under the bilinear pairing:

$$
(\varphi, \phi)=(\varphi(0), \phi(0))-\int_{-1}^{0} \int_{\xi=0}^{\theta} \varphi(\xi-\theta) \mathrm{d} \eta_{1}(\theta, \tilde{\tau}, n) \phi(\xi) \mathrm{d} \xi
$$

where $\phi \in C_{1}, \varphi \in C_{1}\left([-1,0], R^{3 *}\right)$. Thus, $A_{\tau}$ and $A_{\tau}^{*}$ are a pair of adjoint operators, and their eigenvalues are $\Upsilon_{n}=\left\{ \pm i \omega_{n} \widetilde{\tau}\right\}$. Let $F$ and $F^{*}$ be the center space, that is, the generalized characteristic space of $A_{\tau}$ and $A_{\tau}^{*}$, respectively. Then, $F^{*}$ is adjoint space of $F$, and $\operatorname{dim} F=\operatorname{dim} F^{*}$. Thus, we have the following lemma.

Lemma 5. Let

$$
\begin{aligned}
& \gamma_{1}=\frac{a_{21}+b_{21} e^{-i \omega_{n} \tilde{\tau}}}{i \omega_{n}-a_{22}}, \\
& \gamma_{2}=\frac{\left(i \omega_{n}-a_{11}\right)\left(i \omega_{n}-a_{22}\right)-a_{12}\left(a_{21}+b_{21} e^{-i \omega_{n} \tilde{\tau}}\right)}{a_{13}\left(i \omega_{n}-a_{22}\right)}, \\
& \gamma_{1}^{*}=-\frac{a_{12}}{i \omega_{n}+a_{22}}, \\
& \gamma_{2}^{*}=\frac{a_{12}\left(a_{21}+b_{21} e^{i \omega_{n} \tilde{\tau}}\right)-\left(i \omega_{n}+a_{11}\right)\left(i \omega_{n}+a_{22}\right)}{\left(i \omega_{n}+a_{22}\right)\left(a_{31}+b_{31} e^{i \omega_{n} \tilde{\tau}}\right)},
\end{aligned}
$$

and then, $p_{1}(\theta)=\left(1, \gamma_{1}, \gamma_{2}\right)^{T} e^{i \omega_{n} \tilde{\tau} \theta}$ and $p_{2}(\theta)=\overline{p_{1}(\theta)}$, $(-1<\theta<0)$ are a basis of $F$ associated with $\Upsilon_{n}$, and $p_{1}^{*}(\xi)=$ $\left(1, \gamma_{1}^{*}, \gamma_{2}^{*}\right) e^{-i \omega_{n} \tilde{\tau} \theta}$ and $p_{2}^{*}(\xi)=\overline{p_{1}^{*}(\xi)},(0 \leq \xi \leq 1)$, are a basis of $F^{*}$ associated with $\Upsilon_{n}$.

Denoting $\Phi=\left(\Phi_{1}, \Phi_{2}\right)$ and $\Psi^{*}=\left(\Psi_{1}^{*}, \Psi_{2}^{*}\right)^{T}$, where

$$
\begin{aligned}
& \Phi_{1}=\frac{p_{1}(\theta)+p_{2}(\theta)}{2}, \\
& \Phi_{2}=\frac{p_{1}(\theta)-p_{2}(\theta)}{2 i}, \quad \theta \in[-1,0], \\
& \Psi_{1}^{*}=\frac{p_{1}^{*}(\xi)+p_{2}^{*}(\xi)}{2}, \\
& \Psi_{1}^{*}=\frac{p_{1}^{*}(\xi)-p_{2}^{*}(\xi)}{2 i}, \quad \xi \in[0,1],
\end{aligned}
$$

and we define that

$$
\left(\Psi^{*}, \Phi\right)=\left(\Psi_{k}^{*}, \Phi_{l}\right)=\left(\begin{array}{ll}
\left(\Psi_{1}^{*}, \Phi_{1}\right) & \left(\Psi_{1}^{*}, \Phi_{2}\right) \\
\left(\Psi_{2}^{*}, \Phi_{1}\right) & \left(\Psi_{2}^{*}, \Phi_{2}\right)
\end{array}\right)
$$

and construct a new basis $\Psi$ for $F^{*}$ by $\Psi=\left(\Psi_{1}, \Psi_{2}\right)^{T}=\left(\Psi^{*}, \Phi\right)^{-1} \Psi^{*}$, so $(\Psi, \Phi)=I$.

Let $f_{n}=\left(\eta_{n}^{1}, \eta_{n}^{2}, \eta_{n}^{3}\right)$, where $\eta_{n}^{1}=(\cos n x, 0,0)^{T}, \eta_{n}^{2}=$ $(0, \cos n x, 0)^{T}$, and $\eta_{n}^{3}=(0,0, \cos n x)^{T}$. Define

$$
\begin{aligned}
v \cdot f_{n} & =v_{1} \eta_{n}^{1}+v_{2} \eta_{n}^{2}+v_{3} \eta_{n}^{3}, \\
v & =\left(v_{1}, v_{2}, v_{3}\right)^{T} \in C_{1}([-1,0], X) .
\end{aligned}
$$

From $\mathrm{Wu}$ [55], the center space of linear equation (71) is given by $P_{C N} \wp$, where

$$
P_{C N} \varphi=\Phi\left(\Psi,\left\langle\varphi, f_{n}\right\rangle\right) \cdot f_{n}, \quad \varphi \in \wp,
$$

and $C_{1}=P_{C N} \wp \oplus P_{S} C_{1} ; P_{S} C_{1}$ denotes the complementary subspace of $P_{C N} \wp$.

According to Hassard et al. [51] and Wu [55], the center space of the linear model of model (68) with $\mu_{2}=0$ is given by $P_{C N} \wp$, where

$$
P_{C N} \wp=\left\{\frac{1}{2}\left(p_{1}(\theta) z+p_{2}(\theta) \bar{z}\right) \cdot f_{n}, z \in C_{1}\right\} .
$$

Hence, the solutions of model (68) are

$$
U_{t}=\frac{1}{2}\left(p_{1}(\theta) z+p_{2}(\theta) \bar{z}\right) \cdot f_{n}+Q(z(t), \bar{z}(t))(\theta),
$$

where $Q(z(t), \bar{z}(t))(\theta)=w(((z+\bar{z}) / 2),(i(z-\bar{z}) / 2), 0)$, $z=x_{1}-i x_{2}$. Therefore, $z$ satisfies

$$
\dot{z}=i \omega_{n} \tilde{\tau} z+g_{1}(z, \bar{z}),
$$

where $g_{1}(z, \bar{z})=\left(\Psi_{1}(0)-i \Psi_{2}(0)\right)\left\langle h\left(U_{t}, 0\right), f_{n}\right\rangle, \Psi \quad(0)=$ $\left(\Psi_{1}(0), \Psi_{2}(0)\right)^{T}$.

Let

$$
\begin{aligned}
& Q(z, \bar{z})=Q_{20} \frac{z^{2}}{z}+Q_{11} z \bar{z}+Q_{02} \frac{\bar{z}^{2}}{z}+\cdots, \\
& g_{1}(z, \bar{z})=\tilde{g}_{20} \frac{z^{2}}{2}+\widetilde{g}_{11} z \bar{z}+\tilde{g}_{02} \frac{\bar{z}^{2}}{z}+\tilde{g}_{21} \frac{z^{2} \bar{z}}{2}+\cdots,
\end{aligned}
$$

and $\left(\kappa_{1}, \kappa_{2}, \kappa_{3}\right)=\Psi_{1}(0)-i \Psi_{2}(0)$. From equations (70) and (85), we can get 


$$
\begin{aligned}
& \tilde{g}_{20}=\frac{\tilde{\tau}}{2}\left\langle\left[a_{14}+\gamma_{1}^{2} a_{15}+\gamma_{1} a_{16}+\gamma_{2} a_{17}\right) \kappa_{1}+\left(a_{24}+\gamma_{1}^{2} a_{25}+\gamma_{1} a_{26}+b_{24} e^{-i 2 \omega_{n} \tilde{\tau}}+\gamma_{1} b_{25} e^{-i \omega_{n} \tilde{\tau}}\right) \kappa_{2}\right. \\
& \left.\left.+\left(a_{34}+\gamma_{2} a_{35}+b_{34} e^{-i 2 \omega_{n} \tilde{\tau}}+\gamma_{2} b_{35} e^{-i \omega_{n} \tilde{\tau}}\right) \kappa_{3}\right] \cos ^{2} n x, \cos n x\right\rangle, \\
& \tilde{g}_{11}=\frac{\widetilde{\tau}}{4}\left\langle\left[\left(2 a_{14}+2 \gamma_{1} \bar{\gamma}_{1} a_{25}+\left(\gamma_{1}+\bar{\gamma}_{1}\right) a_{16}+\left(\gamma_{2}+\bar{\gamma}_{2}\right) a_{17}\right) \kappa_{1}+\left(2 a_{24}+2 \gamma_{1} \bar{\gamma}_{1} a_{25}+\left(\gamma_{1}+\bar{\gamma}_{1}\right) a_{26}+2 b_{24}+\left(\gamma_{1} e^{i \omega_{n} \tilde{\tau}}+\bar{\gamma}_{1} e^{-i \omega_{n} \tilde{\tau}}\right) b_{25}\right) \kappa_{2}\right)\right. \\
& \left.\left.+\left(2 a_{34}+\left(\gamma_{2}+\bar{\gamma}_{2}\right) a_{35}+2 b_{34}+\left(\gamma_{2} e^{i \omega_{n} \tau}+\bar{\gamma}_{2} e^{-i \omega_{n} \tilde{\tau}}\right) b_{35}\right) \kappa_{3}\right] \cos ^{2} n x, \cos n x\right\rangle, \\
& \tilde{g}_{21}=\tilde{\tau}\left\{\left\langle\left[a_{14}\left(Q_{20}^{(1)}(0)+2 Q_{11}^{(1)}(0)\right)+a_{15}\left(Q_{20}^{(2)}(0)+2 Q_{11}^{(2)}(0)\right)+a_{16}\left(\gamma_{1} Q_{11}^{(1)}(0)+\frac{1}{2} \bar{\gamma}_{1} Q_{20}^{(1)}(0)+Q_{11}^{(2)}(0)+\frac{1}{2} Q_{20}^{(2)}(0)\right)\right\rangle\right.\right. \\
& \left.+a_{17}\left(\gamma_{2} Q_{11}^{(1)}(0)+\frac{1}{2} \bar{\gamma}_{2} Q_{20}^{(1)}(0)+Q_{11}^{(3)}(0)+\frac{1}{2} Q_{20}^{(3)}(0)\right)\right] \cos n x, \cos n x \kappa_{1}+\left[a_{24}\left(Q_{20}^{(1)}(0)+2 Q_{11}^{(1)}(0)\right)\right. \\
& +a_{25}\left(Q_{20}^{(2)}(0)+2 Q_{11}^{(2)}(0)\right)+a_{26}\left(\gamma_{1} Q_{11}^{(1)}(0)+\frac{1}{2} \bar{\gamma}_{1} Q_{20}^{(1)}(0)+Q_{11}^{(2)}(0)+\frac{1}{2} Q_{20}^{(2)}(0)\right)+b_{24}\left(2 e^{-i \omega_{n} \tau} Q_{11}^{(1)}(-1)+e^{i \omega_{n} \tau} Q_{20}^{(1)}(-1)\right) \\
& \left.\left.+b_{25}\left(\gamma_{1} Q_{11}^{(1)}(-1)+\frac{1}{2} \bar{\gamma}_{1} Q_{20}^{(1)}(-1)+e^{-i \omega_{n} \tau} Q_{11}^{(2)}(0)+\frac{1}{2} e^{i \omega_{n} \tau} Q_{20}^{(2)}(0)\right)\right] \cos n x, \cos n x\right\rangle \kappa_{2}+\left\langle\left[ a_{34}\left(Q_{20}^{(1)}(0)+2 Q_{11}^{(1)}(0)\right)\right.\right. \\
& +a_{35}\left(\gamma_{2} Q_{11}^{(1)}(0) 2 Q_{11}^{(1)}(0)+\frac{1}{2} \bar{\gamma}_{2} Q_{20}^{(1)}(0)+Q_{11}^{(3)}(0)+\frac{1}{2} Q_{20}^{(3)}(0)\right)+b_{34}\left(2 e^{-i \omega_{n} \tau} Q_{11}^{(1)}(-1)+e^{i \omega_{n} \tau} Q_{20}^{(1)}(-1)\right) \\
& \left.\left.+b_{35}\left(\gamma_{2} Q_{11}^{(1)}(-1)+\frac{1}{2} \bar{\gamma}_{2} Q_{20}^{(1)}(-1)+e^{-i \omega_{n} \tau} Q_{11}^{(3)}(0)+\frac{1}{2} e^{i \omega_{n} \tau} Q_{20}^{(3)}(0)\right) \cos n x, \cos n x\right\rangle \kappa_{3}\right\},
\end{aligned}
$$

where $n \in N_{0}$. It is obvious that $\int_{0}^{\pi} \cos ^{3}(n x) \mathrm{d} x=0$ and $\tilde{g}_{02}=\tilde{\tilde{g}}_{20}$. When $n=1,2, \ldots$, we have $\tilde{g}_{20}=\tilde{g}_{11}=\tilde{g}_{02}=0$.

When $n=0$, we obtain

$$
\begin{aligned}
\tilde{g}_{20}= & \frac{\tilde{\tau}}{2}\left[\left(a_{14}+\gamma_{1}^{2} a_{15}+\gamma_{1} a_{16}+\gamma_{2} a_{17}\right) \kappa_{1}+\left(a_{24}+\gamma_{1}^{2} a_{25}+\gamma_{1} a_{26}+b_{24} e^{-i 2 \omega_{n} \tilde{\tau}}+\gamma_{1} b_{25} e^{-i \omega_{n} \tilde{\tau}}\right) \kappa_{2}\right. \\
& \left.+\left(a_{34}+\gamma_{2} a_{35}+b_{34} e^{-i 2 \omega_{n} \tilde{\tau}}+\gamma_{2} b_{35} e^{-i \omega_{n} \tilde{\tau}}\right) \kappa_{3}\right], \\
\tilde{g}_{11}= & \frac{\widetilde{\tau}}{4}\left[\left(2 a_{14}+2 \gamma_{1} \bar{\gamma}_{1} a_{15}+\left(\gamma_{1}+\bar{\gamma}_{1}\right) a_{16}+\left(\gamma_{2}+\bar{\gamma}_{2}\right) a_{17}\right) \kappa_{1}+\left(2 a_{24}+2 \gamma_{1} \bar{\gamma}_{1} a_{25}\right)\right. \\
& \left.\left.\left.+\left(\gamma_{1}+\bar{\gamma}_{1}\right) a_{26}+2 b_{24}+\left(\gamma_{1} e^{i \omega_{n} \tau}+\bar{\gamma}_{1} e^{-i \omega_{n} \tilde{\tau}}\right) b_{25}\right) \kappa_{2}+\left(2 a_{34}+\left(\gamma_{2}+\bar{\gamma}_{2}\right) a_{35}+2 b_{34}+\left(\gamma_{2} e^{i \omega_{n} \tilde{\tau}}+\bar{\gamma}_{2} e^{-i \omega_{n} \tilde{\tau}}\right) b_{35}\right) \kappa_{3}\right)\right],
\end{aligned}
$$

and $Q_{20}(0), Q_{20}(-1), Q_{11}(0)$, and $Q_{11}(-1)$ appear in $g_{21}$. From equation (84), we have

$$
\begin{gathered}
\dot{Q}(z, \bar{z})=Q_{20} z \dot{z}+Q_{11} \dot{z} \bar{z}+Q_{11} z \dot{\bar{z}}+Q_{02} \bar{z} \overline{\dot{z}}+\cdots, \\
A_{\tau} Q=A_{\tau} Q_{20} \frac{z^{2}}{2}+A_{\tau} Q_{11} z \bar{z}+A_{\tau} Q_{02} \frac{\bar{z}^{2}}{2}+\cdots .
\end{gathered}
$$

Therefore, according to $\mathrm{Wu}$ [55], we know that $Q(z(t), \bar{z}(t))$ satisfies

$$
\dot{Q}=A_{\tau} Q+G(z, \bar{z}),
$$

where

$$
G(z, \bar{z})=G_{20} \frac{z^{2}}{2}+G_{11} z \bar{z}+G_{02} \frac{\bar{z}^{2}}{2}+\cdots=X_{0} h\left(U_{t}, 0\right)-\Phi\left(\Psi,\left\langle X_{0} h\left(U_{t}, 0\right), f_{n}\right\rangle\right) \cdot f_{n},
$$


where the function $X_{0}$ defined by $X_{0}(0)=I$ and $X_{0}(\theta)=$ $0,-1 \leq \theta<0$, and $G_{i j} \in P_{S} \wp(i+j=2)$.

According to (82) and (88)-(90), we have

$$
\left\{\begin{array}{l}
\left(2 i \omega_{n} \tilde{\tau}-A_{\tilde{\tau}}\right) Q_{20}=G_{20}, \\
-A_{\tilde{\tau}} Q_{11}=G_{11}, \\
\left(-2 i \omega_{n} \widetilde{\tau}-A_{\tilde{\tau}}\right) Q_{02}=G_{02} .
\end{array}\right.
$$

Since $A_{\tau}$ has a pair of purely imaginary characteristic roots, which are $\pm i \omega_{n} \widetilde{\tau}$, equation (91) has the unique solution $Q_{i j}$.

From equation (92), we obtain that, for $\theta \in[-1,0)$,

$$
\begin{aligned}
G(z, \widetilde{z}) & =-\Phi(\theta) \Psi(0)\left\langle h\left(U_{t}, 0\right), f_{n}\right\rangle \cdot f_{n} \\
& =-\frac{1}{2} \widetilde{\tau}\left[\left(p_{1}(\theta) \widetilde{g}_{20}+\overline{\widetilde{g}_{02}} \overline{p_{1}(\theta)}\right) \cdot f_{n} \frac{z^{2}}{2}+\left(p_{1}(\theta) \widetilde{g}_{11}+\overline{\widetilde{g}_{11}} \overline{p_{1}(\theta)}\right) \cdot f_{n} z \bar{z}+\left(p_{1}(\theta) \widetilde{g}_{02}+\overline{\widetilde{g}_{20}} \overline{p_{1}(\theta)}\right) \cdot f_{n} \frac{\bar{z}^{2}}{2}\right]+\cdots
\end{aligned}
$$

Therefore, by comparing the coefficients of equations (91) and (93) and noticing that

$$
G(z, \tilde{z})(0)=h\left(U_{t}, 0\right)-\Phi\left(\Psi,\left\langle h\left(U_{t}, 0\right), f_{n}\right\rangle\right) \cdot f_{n}=h\left(U_{t}, 0\right)-\Phi(\theta) \Psi(0)\left\langle h\left(U_{t}, 0\right), f_{n}\right\rangle \cdot f_{n}
$$

we obtain that

$$
\begin{aligned}
& \int-\frac{1}{2} \widetilde{\tau}\left(p_{1}(\theta) \widetilde{g}_{20}+\overline{\widetilde{g}}_{20} \overline{p_{1}(\theta)}\right) \cos (n x), \quad \theta \in[-1,0),
\end{aligned}
$$

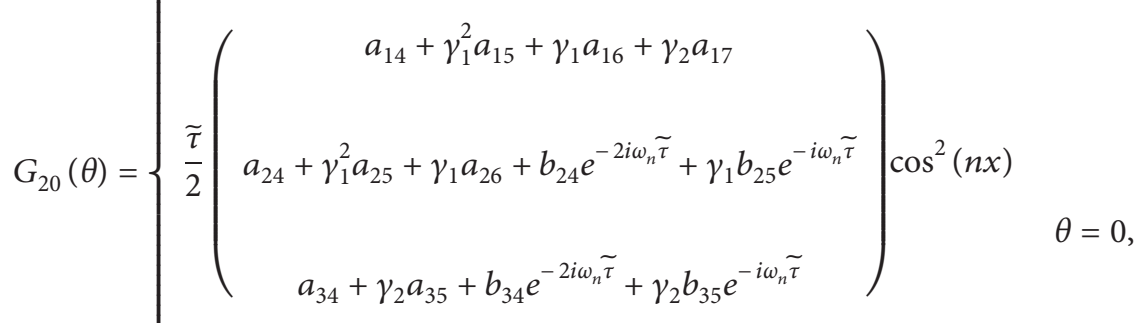

$$
\begin{aligned}
& -\frac{1}{2} \widetilde{\tau}\left(p_{1}(\theta) \widetilde{g}_{20}+\overline{\widetilde{g}}_{20} \overline{p_{1}(\theta)}\right) \cos (n x),
\end{aligned}
$$

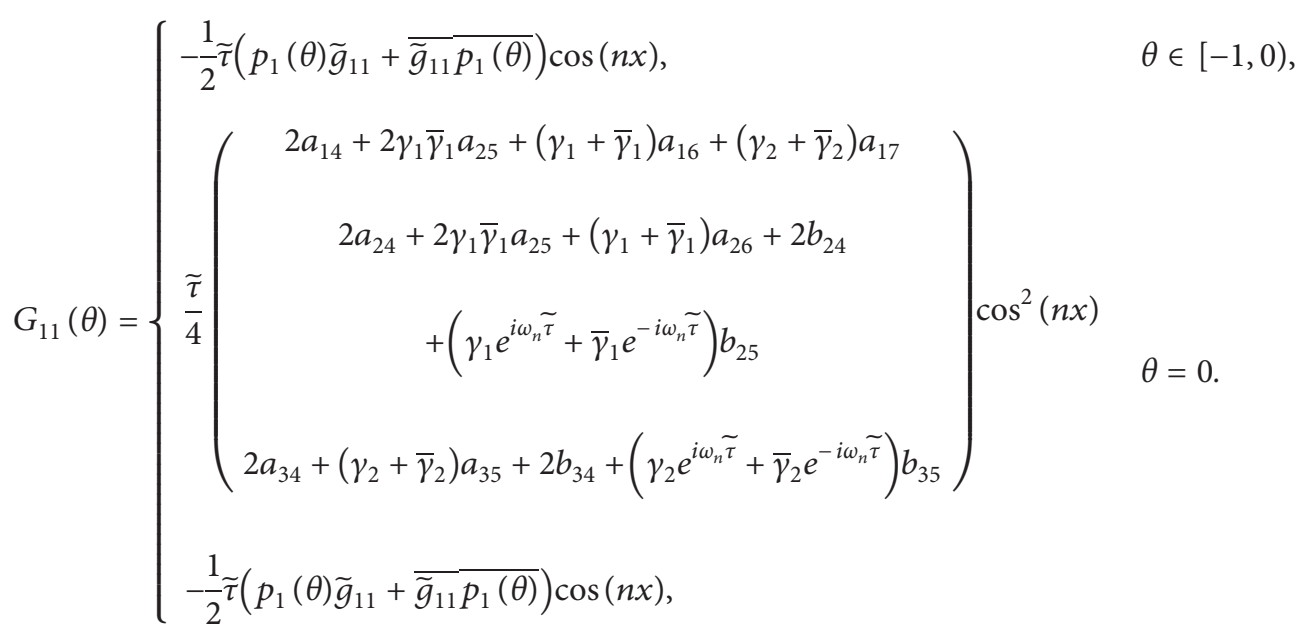


Next, we compute $Q_{20}(0), Q_{20}(-1), Q_{11}(0)$, and $Q_{11}(-1)$ by the variation-of-constants method. According to equations (92)-(96), we have

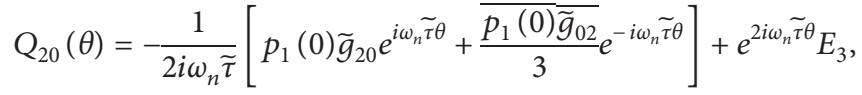

$$
\begin{aligned}
& Q_{11}(\theta)=\frac{1}{2 i \omega_{n} \tilde{\tau}}\left[p_{1}(0) \widetilde{g}_{11} e^{i \omega_{n} \tilde{\tau} \theta}-\overline{p_{1}(0)} \overline{\tilde{g}}_{11} e^{-i \omega_{n} \tilde{\tau} \theta}\right]+E_{4} \text {. }
\end{aligned}
$$

Furthermore, for $Q_{i j} \in C_{1}$, we have

$$
\begin{aligned}
& Q_{20}(0)=-\frac{1}{2 i \omega_{n} \widetilde{\tau}}\left(p_{1}(0) \widetilde{g}_{20}+\frac{\overline{p_{1}(0)} \widetilde{\tilde{g}}_{02}}{3}\right)+E_{3}, \\
& Q_{11}(0)=\frac{1}{2 i \omega_{n} \widetilde{\tau}}\left(p_{1}(0) \widetilde{g}_{11}-\overline{p_{1}(0)} \widetilde{\tilde{g}}_{11}\right)+E_{4},
\end{aligned}
$$

where $E_{3}=\left(E_{3}^{(1)}, E_{3}^{(2)}, E_{3}^{(3)}\right)^{T} \in R^{3}$ and $E_{4}=\left(E_{4}^{(1)}, E_{4}^{(2)}\right.$, $\left.E_{4}^{(3)}\right)^{T} \in R^{3}$ are given as follows, respectively:

$$
\begin{gathered}
E_{3}=\frac{1}{2}\left(\begin{array}{ccc}
2 i \omega_{n}-a_{11} & -a_{12} & -a_{13} \\
-a_{21}-b_{21} e^{-2 i \omega_{n} \tilde{\tau}} & 2 i \omega_{n}-a_{22} & 0 \\
-a_{31}-b_{31} e^{-2 i \omega_{n} \tau} & 0 & 2 i \omega_{n}
\end{array}\right)^{-1} \times\left(\begin{array}{c}
a_{14}+\gamma_{1}^{2} a_{15}+\gamma_{1} a_{16}+\gamma_{2} a_{17} \\
a_{24}+\gamma_{1}^{2} a_{25}+\gamma_{1} a_{26}+b_{24} e^{-2 i \omega_{n} \tilde{\tau}}+\gamma_{1} b_{25} e^{-i \omega_{n} \tilde{\tau}} \\
a_{34}+\gamma_{2} a_{35}+b_{34} e^{-2 i \omega_{n} \tilde{\tau}}+\gamma_{2} b_{35} e^{-i \omega_{n} \tilde{\tau}}
\end{array}\right) \cos ^{2}(n x), \\
E_{4}=\frac{1}{4}\left(\begin{array}{ccc}
-a_{11} & -a_{12} & -a_{13} \\
-a_{21}-b_{21} & -a_{22} & 0 \\
-a_{31}-a_{31} & 0 & 0
\end{array}\right)^{-1} \times\left(\begin{array}{c}
2 a_{14}+2 \gamma_{1} \bar{\gamma}_{1} a_{25}+\left(\gamma_{1}+\bar{\gamma}_{1}\right) a_{16}+\left(\gamma_{2}+\bar{\gamma}_{2}\right) a_{17} \\
2 a_{24}+2 \gamma_{1} \bar{\gamma}_{1} a_{25}+\left(\gamma_{1}+\bar{\gamma}_{1}\right) a_{26}+2 b_{24}+\left(\gamma_{1} e^{i \omega_{n} \tilde{\tau}}+\bar{\gamma}_{1} e^{-i \omega_{n} \tilde{\tau}}\right) b_{25} \\
2 a_{34}+\left(\gamma_{2}+\bar{\gamma}_{2}\right) a_{35}+2 b_{34}+\left(\gamma_{2} e^{i \omega_{n} \tau}+\bar{\gamma}_{2} e^{-i \omega_{n} \tilde{\tau}}\right) b_{35}
\end{array}\right) \cos ^{2}(n x) .
\end{gathered}
$$

In the following, we can compute the following quantities that determine the properties of bifurcating periodic solutions:

$$
\left\{\begin{array}{l}
c_{2}(0)=\frac{i}{2 \omega_{n} \tilde{\tau}}\left(\tilde{g}_{20} \widetilde{g}_{11}-2\left|\widetilde{g}_{11}\right|^{2}-\frac{\left|\tilde{g}_{02}\right|^{2}}{3}\right)+\frac{\widetilde{g}_{21}}{2}, \\
v_{2}=-\frac{\operatorname{Re}\left\{c_{2}(0)\right\}}{\operatorname{Re}\left\{\lambda^{\prime}(\tilde{\tau})\right\}} \\
\varrho_{2}=2 \operatorname{Re}\left\{c_{2}(0)\right\} \\
T_{2}=-\frac{\operatorname{Im}\left\{c_{2}(0)\right\}+v_{2} \operatorname{Im}\left\{\lambda^{\prime}(\tilde{\tau})\right\}}{\omega_{n} \widetilde{\tau}} .
\end{array}\right.
$$

Then, by the Hopf bifurcation theory [51], we can obtain the following result.

Theorem 12. Assume that Theorem 9 or Theorem 10 holds, the following results on equation (100) are true.

(1) $v_{2}$ determines the Hopf bifurcation direction. If $v_{2}>0(<0)$, then the Hopf bifurcation is forward (backward) and the bifurcating periodic solutions exist for $\tau>\tilde{\tau}$.

(2) $\varrho_{2}$ determines the stability of bifurcating periodic solutions. If $\varrho_{2}<0(>0)$, then the periodic solutions are stable (unstable).
(3) $T_{2}$ determines the period of the bifurcating periodic solutions. If $T_{2}>0(<0)$, then the period increases (decreases).

\section{Numerical Simulation}

In this section, we present some numerical results of system (3) to support and extend our theoretical results which have been established in the previous sections. The spatial domain is $[0, \pi]$. Next, we simulate those results by using the software Matlab.

First, we simulate system (4). We assume that $P(0)=$ $9, Z_{1}(0)=20$, and $Z_{2}(0)=1$ and choose the following parameter values: $r=11, K=25, \mu_{1}=10, \mu_{2}=9, \alpha_{1}=10, \alpha_{2}=$ $20, \beta_{1}=5, \beta_{2}=5, \rho_{1}=1, \rho_{2}=1.400, g_{1}=1, g_{2}=1.110$, and $s=0.700$ so that $\bar{P}>\left(\left(g_{1} \alpha_{1}\right) /\left(\beta_{1}-\rho_{1}-g_{1}\right)\right), r(K-\bar{P})$ $\left(\beta_{1}-\rho_{1}\right) \bar{P}>K g_{1} \mu_{1} \bar{Z}_{1}$, and $\left(\mu_{1} \bar{Z}_{1} /\left(\left(\alpha_{1}+\bar{P}+s \bar{Z}_{1}\right)^{2}\right)\right)+$ $\left(\mu_{2} \bar{P} /\left(\left(\alpha_{2}+\bar{P}\right)^{2}\right)\right)<(\bar{P} / K)$. According to Theorem 4 , we can get that system (4) has the coexistence equilibrium $E^{*}(8.916,23.924,1.184)$ and $E^{*}$ is locally asymptotically stable, see Figure 1.

For system (4), we consider $s$ as the bifurcation parameter under $P(0)=6, Z_{1}(0)=2$, and $Z_{2}(0)=1$. We select a set of parameters: $r=1, K=20, \mu_{1}=5, \mu_{2}=6, \alpha_{1}=$ $6, \alpha_{2}=10, \beta_{1}=4, \beta_{2}=5, \rho_{1}=1, \rho_{2}=1.400, g_{1}=1$, and $g_{2}=1.110$. By simple calculation, we can know that $s^{*}=2.199$. Thus, $E^{*}(4.578,1.944,0.121)$ is locally asymptotically stable when $s=1.500<s^{*}$, see Figure 2. When $s=11>s^{*}, E^{*}$ is unstable, see Figure 3 .

Next, we choose the values of parameters: $r=1, K=$ $20, \mu_{1}=5, \mu_{2}=6, \alpha_{1}=6, \alpha_{2}=10, \beta_{1}=4, \beta 2=5, \rho_{1}=1, \rho_{2}$ 


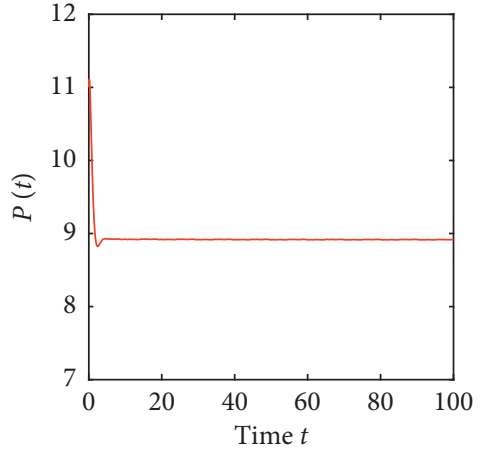

(a)

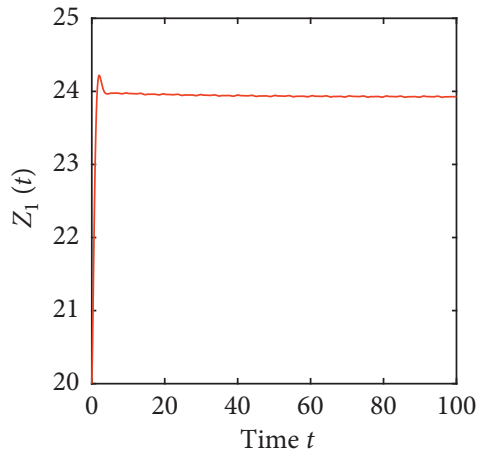

(b)

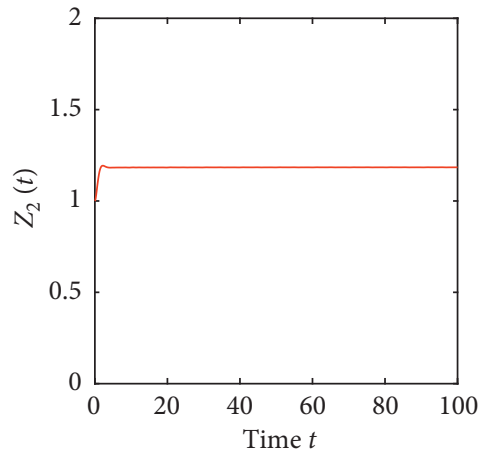

(c)

FIgURE 1: The coexistence equilibrium $E^{*}$ of system (4) is locally asymptotically stable when $\tau=0$. (a) $P(t)$; (b) $Z_{1}(t)$; (c) $Z_{2}(t)$.

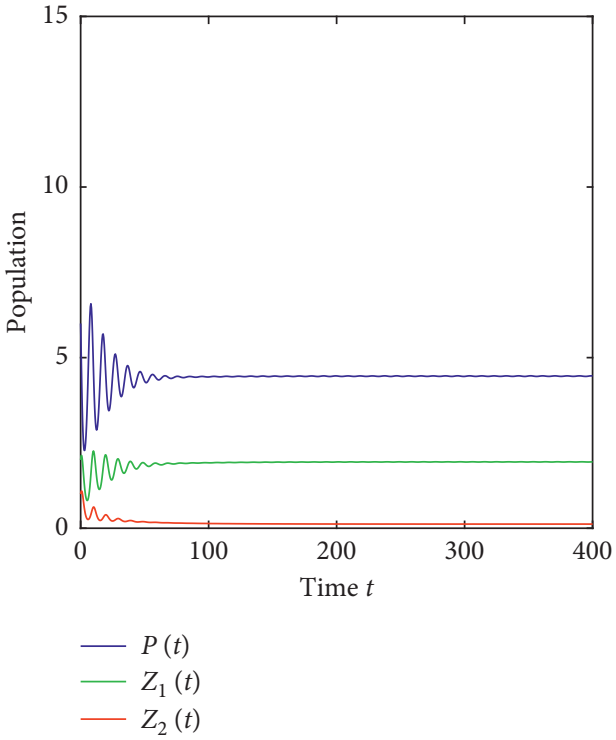

(a)

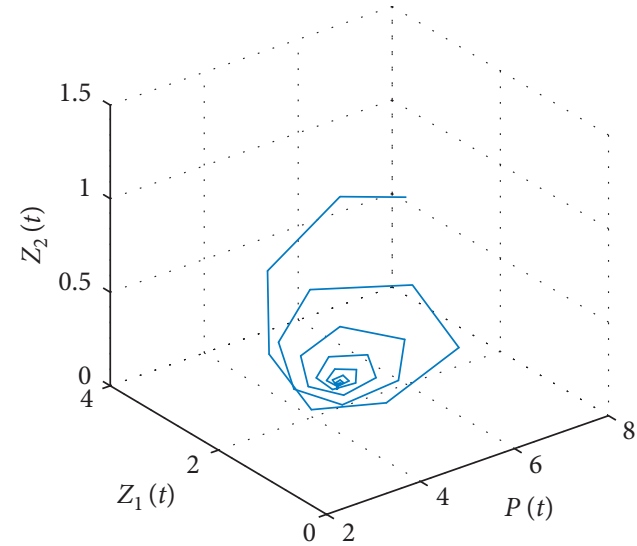

(b)

Figure 2: The coexistence equilibrium $E^{*}(4.458,1.944,0.121)$ of system (4) is locally asymptotically stable when $s=1.500<s^{*}=2.199$. (a) Dynamical response of population. (b) Phase portrait.

$=1.400, g_{1}=1, g_{2}=1.110$, and $s=4$. We can get $\omega_{1}^{0}=$ 3.433 and $\tau_{0}=1.830$ when $\tau \neq 0$. According to Theorem 6 , we obtain that the coexistence equilibrium $E^{*}(4.458$, $0.729,1.216)$ is locally asymptotically stable when $\tau=2<\tau_{0}$, see Figure 4. Then, we select the values of $\tau$ as $\tau=3.500>\tau_{0}$ and get that $c_{1}(0) \approx-1.573-$ $8.542 i<0, \nu_{1} \approx 0.458>0, \quad \varrho_{1} \approx-3.145<0$, and $T_{1} \approx$ $0.711>0$ by using formula (51). From Theorem 2, we know that system (21) undergoes Hopf bifurcation and the bifurcation direction is forward when $\tau$ passes through its critical value $\tau_{0}$. In addition, system (21) has a stable bifurcation periodic solution, and the period of the bifurcating periodic solutions is increasing. From Figure 5, we know that the coexistence equilibrium $E^{*}(4.458,0.729,1.216)$ is destabilized through a Hopf bifurcation.
Finally, we choose a new set of parameter values to simulate system (3). $d_{1}=0.500, d_{2}=0.500, d_{3}=0.400, r=$ $1, K=20, \mu_{1}=5, \mu_{2}=6, \alpha_{1}=6, \alpha_{2}=10, \beta_{1}=4, \beta_{2}=5, \rho_{1}=$ $1, \rho_{2}=1.400, g_{1}=1, g_{2}=1.110$, and $s=6$. From Theorem 7 , we can get that the coexistence equilibrium $E^{*}(4.458,0.486$, 1.435) is locally asymptotically stable when $\tau=0$, see Figure 6.

Through simple calculation, we obtain that $\omega_{2}^{0}=0.348$, and $\tau_{0}^{0}=8.640$. According to Theorem 10, we know that $E^{*}$ is locally asymptotically stable when $\tau=8<\tau_{0}^{0}=8.640$, see Figure 7. Then, we choose the value of $\tau=15>\tau_{0}^{0}=8.640$ and obtain that $c_{2}(0) \approx-213-345 i<0, \quad v_{2} \approx 6120.800$ $>0, \varrho_{2} \approx-4253.900<0$, and $T_{2} \approx 660.823>0$. From Theorem 11, we know that system (3) undergoes a forward Hopf bifurcation when $\tau$ across its critical value $\tau_{0}^{0}=8.640$. System (3) has stable bifurcating periodic solutions, and the period of the bifurcating periodic solutions is increasing. In addition, we 


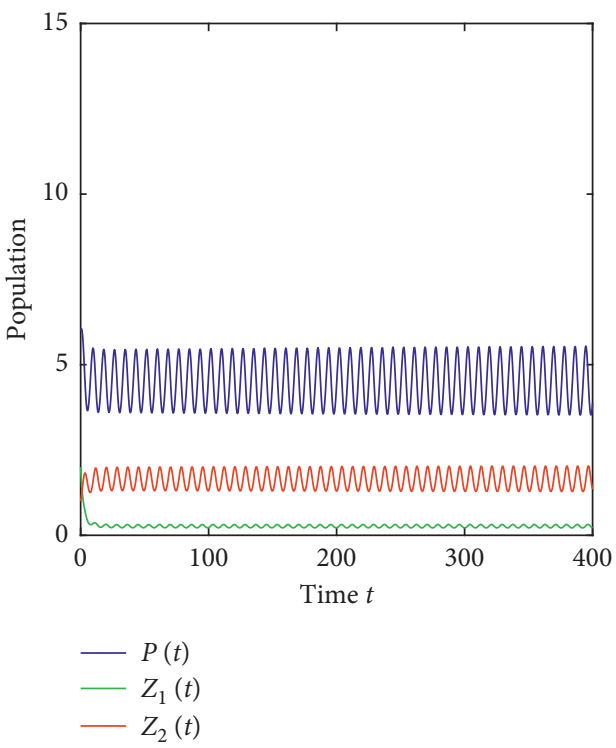

(a)

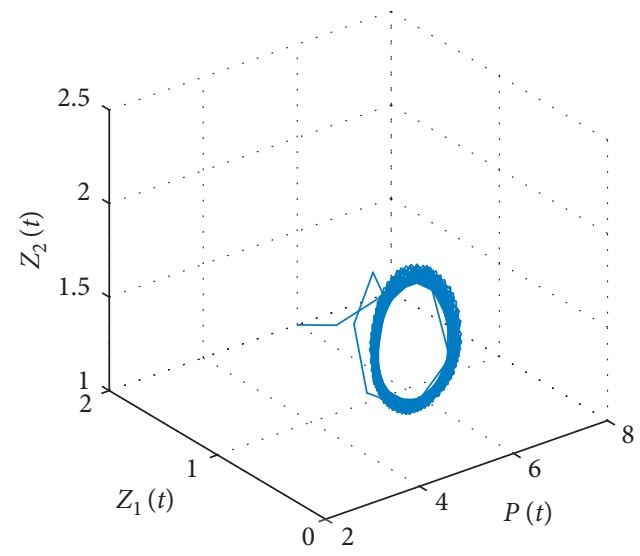

(b)

Figure 3: The coexistence equilibrium $E^{*}$ of system (4) is destabilized through a Hopf bifurcation when $s=11>s^{*}=2.199$. (a) Dynamical response of population. (b) Phase portrait.

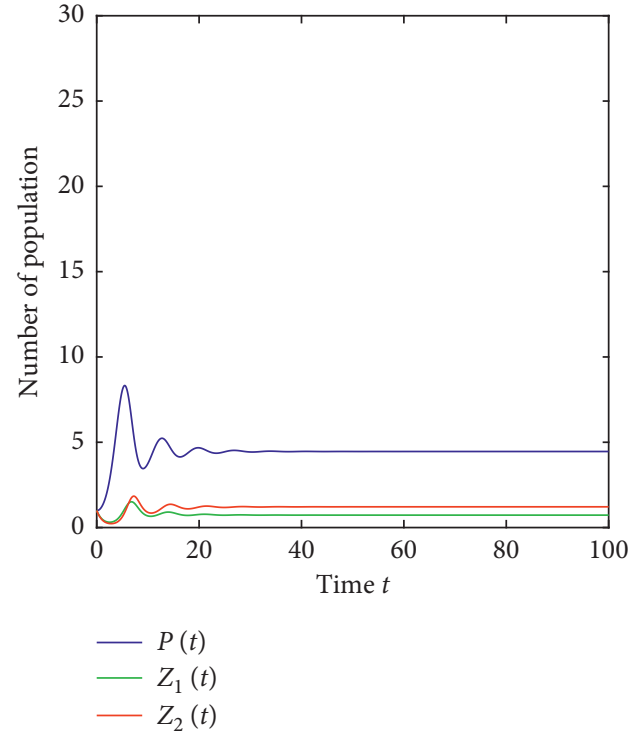

(a)

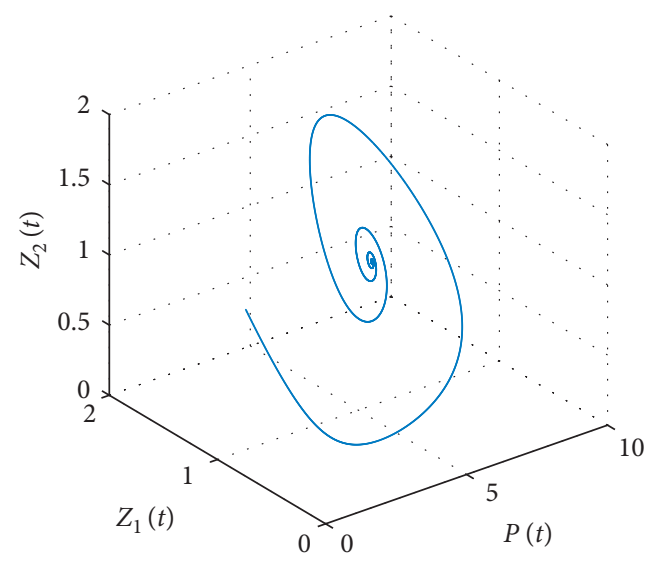

(b)

Figure 4: The coexistence equilibrium $E^{*}(4.458,0.729,1.216)$ of model $(21)$ is locally asymptotically stable when $\tau=1<\tau_{0}=1.830$. (a) Dynamical response of population. (b) Phase portrait. 


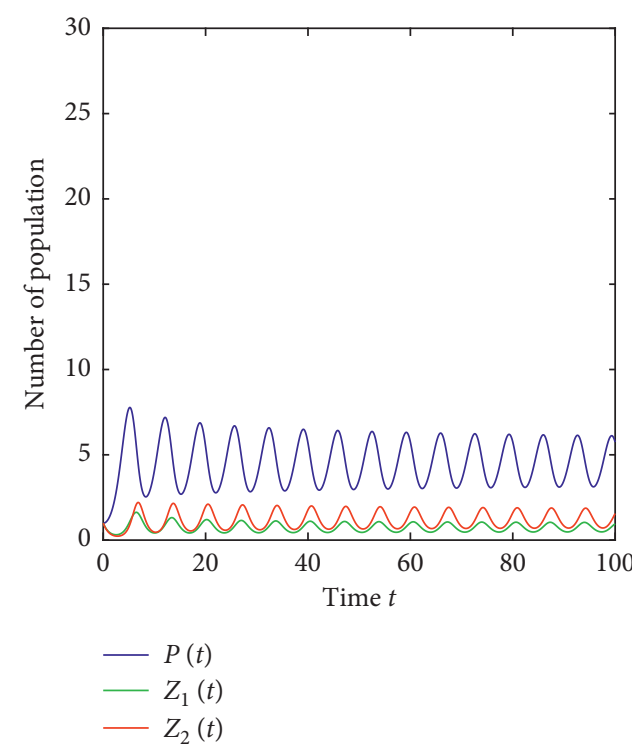

(a)

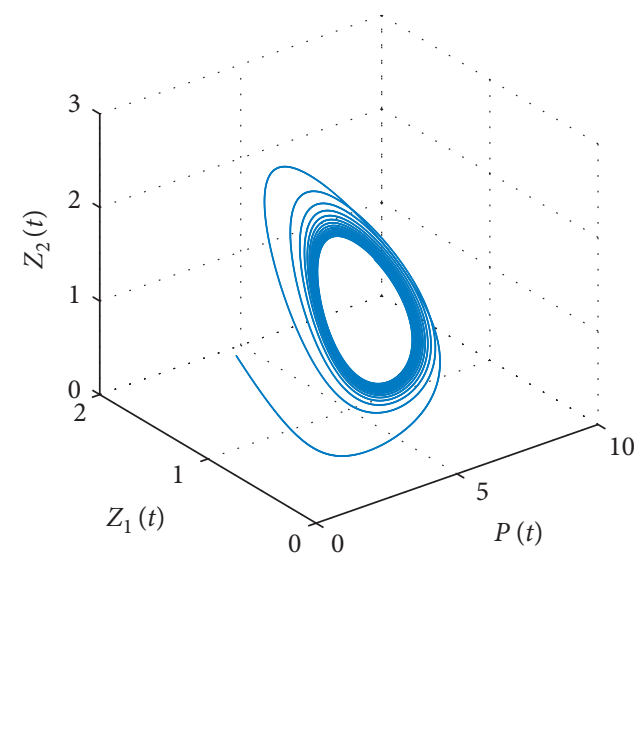

(b)

FIGURE 5: When $\tau=3.500>\tau_{0}=1.830$, Hopf bifurcation takes place at the coexistence equilibrium $E^{*}(4.458,0.729,1.216)$ of system $(21)$. (a) Dynamical response of population. (b) Phase portrait.

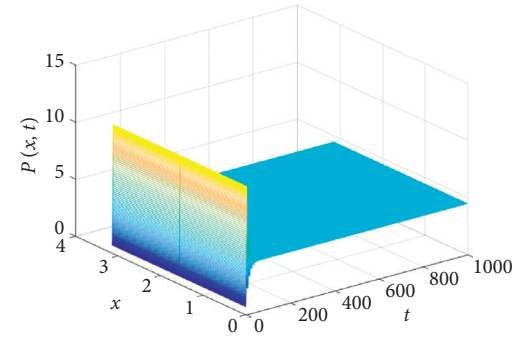

(a)

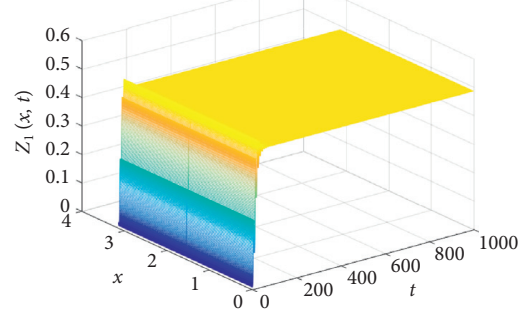

(b)

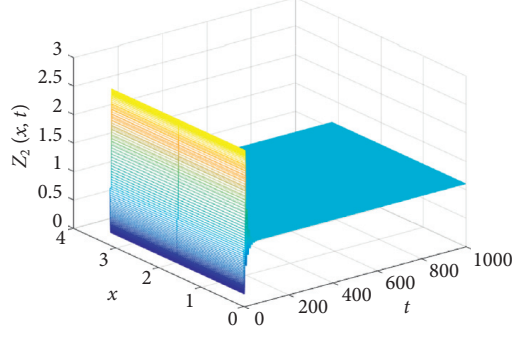

(c)

Figure 6: The coexistence equilibrium $E^{*}(4.458,0.486,1.435)$ of system (3) is locally asymptotically stable when $\tau=0$. (a) $P(t)$; (b) $Z_{1}(t)$; (c) $Z_{2}(t)$.

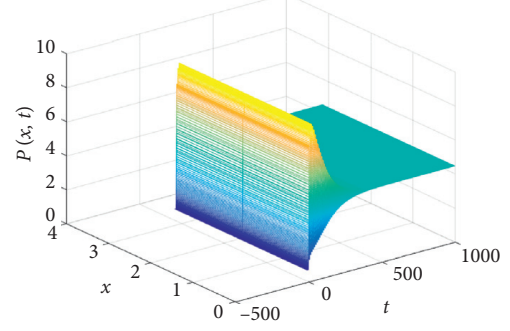

(a)

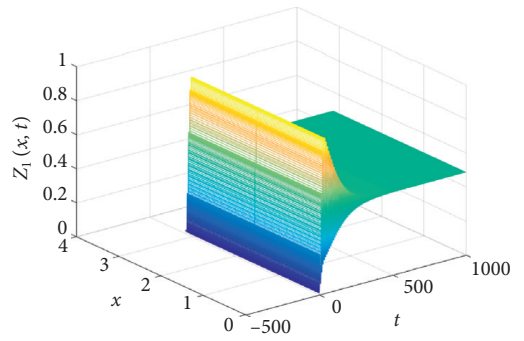

(b)

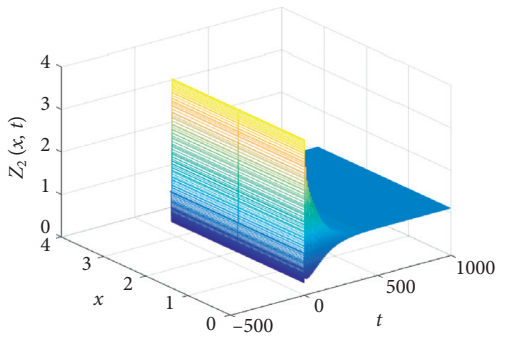

(c)

Figure 7: The coexistence equilibrium $E^{*}(4.458,0.486,1.435)$ of system $(3)$ is locally asymptotically stable when $\tau=8<\tau_{0}^{0}=8.640$. (a) $P(t)$; (b) $Z_{1}(t)$; (c) $Z_{2}(t)$. 


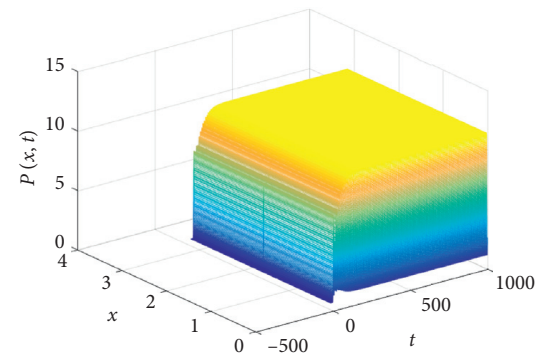

(a)

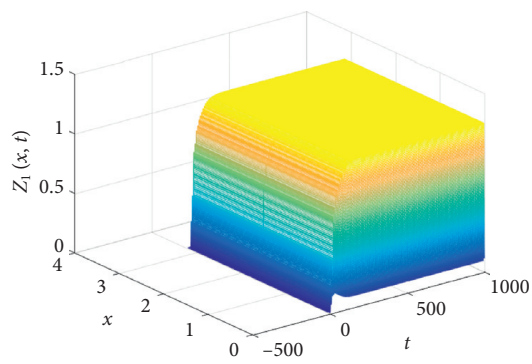

(b)

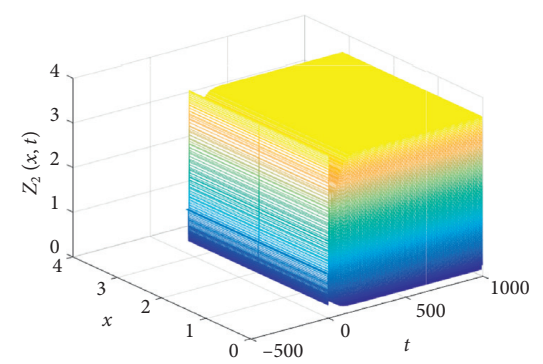

(c)

Figure 8: The coexistence equilibrium $E^{*}(4.458,0.486,1.435)$ of system (3) is destabilized through a Hopf bifurcation when $\tau=15>\tau_{0}^{0}=8.640$. (a) $P(t)$; (b) $Z_{1}(t)$; (c) $Z_{2}(t)$.

see from Figure 8 that the coexistence equilibrium $E^{*}(4.458,0.486,1.435)$ is destabilized by a Hopf bifurcation.

\section{Conclusions and Discussions}

In this paper, we have proposed a reaction-diffusion model with time delay and two kinds of functional response incorporating three plankton populations, where the functional responses of two zooplankton to phytoplankton are different. Because there is interference between some zooplankton in freshwater lakes or oceans, so we assume that the functional response of zooplankton $Z_{1}(t, x)$ is Beddington-DeAnglis functional response. We verify that the degree of mutual interference $s$ can produce Hopf bifurcation using numerical simulation; thus, we can change the value of this parameter $s$ to control the explosion of plankton. Then, we consider time delay $\tau$ as bifurcation parameters to study the dynamics of systems (21) and (3). We discuss the existence of the Hopf bifurcation when $\tau$ passes through the critical values $\tau_{0}$ or $\tau_{0}^{0}$. In addition, we get that properties of Hopf bifurcation by utilizing the normal form theory and the center manifold theorem. By analysis, we know that systems (3) and (21) are stable at the coexistence equilibrium $E^{*}$ when $\tau<\tau_{0}^{0}$ and $\tau<\tau_{0}$, respectively. But systems (3) and (21) are unstable at the coexistence equilibrium $E^{*}$ when $\tau>\tau_{0}^{0}$ and $\tau>\tau_{0}$, respectively. These results indicate that if time delay $\tau$ required for mature phytoplankton to produce toxins is small enough, then the densities of phytoplankton $P$ and zooplankton $Z_{1}$ and $Z_{2}$ will tend to constant; but if $\tau$ is too large, systems (3) and (21) will have periodic solutions, which means that the densities of phytoplankton $P$ and zooplankton $Z_{1}$ and $Z_{2}$ will change periodically. Through analysis, we find that the size of the diffusion coefficient does not change the stability of the system.

In order to make the model more realistic, we will consider linear harvesting of zooplankton, such as $q_{1} E Z_{1}$ and $q_{1} E Z_{1}$, where $q_{1}$ and $q_{2}$ are the catchability coefficients of zooplankton $Z_{i}$ (i=1 and 2), respectively, and $E$ represents the harvesting effort. The model is as follows:

$$
\begin{cases}\frac{\partial P(t, x)}{\partial t}=d_{1} \Delta P(t, x)+r P(t, x)\left(1-\frac{P(t, x)}{K}\right)-\frac{\mu_{1} P(t, x) Z_{1}(t, x)}{\alpha_{1}+P(t, x)+s Z_{1}(t, x)}-\frac{\mu_{2} P(t, x) Z_{2}(t, x)}{\alpha_{2}+P(t, x)}, & t>0 \text { and } x \in(0, \pi), \\ \frac{\partial Z_{1}(t, x)}{\partial t}=d_{2} \Delta Z_{1}(t, x)+\frac{\beta_{1} P(t, x) Z_{1}(t, x)}{\alpha_{1}+P(t, x)+s Z_{1}(t, x)}-g_{1} Z_{1}(t, x) & t>0 \text { and } x \in(0, \pi), \\ -q_{1} E Z_{1}(t, x)-\frac{\rho_{1} P(t-\tau, x) Z_{1}(t, x)}{\alpha_{1}+P(t-\tau, x)+s Z_{1}(t, x)}, & t>0 \text { and } x \in(0, \pi), \\ \frac{\partial Z_{2}(t, x)}{\partial t}=d_{3} \Delta Z_{2}(t, x)+\frac{\beta_{2} P(t, x) Z_{2}(t, x)}{\alpha_{2}+P(t, x)}-\frac{\rho_{2} P(t-\tau, x) Z_{2}(t, x)}{\alpha_{2}+P(t-\tau, x)}-g_{2} Z_{2}(t, x)-q_{2} E Z_{2}(t, x), & t>0 \text { and } x=0 \text { and } \pi, \\ \frac{\partial P(t, x)}{\partial x}=\frac{\partial Z_{1}(t, x)}{\partial x}=\frac{\partial Z_{2}(t, x)}{\partial x}=0, & t \in[-\tau, 0] \text { and } x \in[0, \pi] . \\ P(t, x)=\varphi_{1}(t, x) \geq 0, Z_{1}(t, x)=\varphi_{2}(t, x) \geq 0, & t\end{cases}
$$

We leave this work for the future. 


\section{Data Availability}

The data used to support the findings of our study are included within the article. You can find the corresponding values of some parameters in the beginning of the "Numerical Simulation," which are taken from the numerical simulation section in Reference [22]. Of course, every reader can freely access the parameter values and data supporting the conclusions of the study in [22].

\section{Conflicts of Interest}

The authors declare that there are no conflicts of interest regarding the publication of this paper.

\section{Acknowledgments}

This work was supported by the National Natural Science Foundation of China (Grant nos. 11661050 and 11861044), National Natural Science Foundation of Gansu Province (Grant no. 20JR10RA156), and HongLiu First-Class Disciplines Development Program of Lanzhou University of Technology.

\section{References}

[1] M. Gao, H. H. Shi, and Z. Z. Li, "Chaos in a seasonally and periodically forced phytoplankton-zooplankton system," Nonlinear Analysis: Real World Applications, vol. 10, no. 3, pp. 1643-1650, 2009.

[2] Y. F. Lv, Y. Z. Pei, S. Gao, and C.G. Li, "Harvesting of a phytoplankton-zooplankton model," Nonlinear Analysis: Real World Applications, vol. 11, no. 5, pp. 3608-3619, 2010.

[3] X. Y. Meng and Y. Q. Wu, "Bifurcation and control in a singular phytoplankton-zooplankton-fish model with nonlinear fish harvesting and taxation," International Journal of Bifurcation and Chaos, vol. 28, no. 3, p. 18500, 2018.

[4] X. W. Yu, S. L. Yuan, and T. Q. Zhang, "Asymptotic properties of stochastic nutrient-plankton food chain models with nutrient recycling," Nonlinear Analysis: Hybrid Systems, vol. 34, pp. 209-225, 2019.

[5] P. Panja, "Plankton population and cholera disease transmission: a mathematical modeling study," International Journal of Bifurcation and Chaos, vol. 30, no. 4, Article ID 2050054, 2020.

[6] Z. C. Jiang, J. T. Dai, and T. Q. Zhang, "Bifurcation analysis of phytoplankton and zooplankton interaction system with two delays," International Journal of Bifurcation and Chaos, vol. 30, no. 3, pp. 331-340, 2020.

[7] T. Liao, H. G. Yu, H. Yu, C. Dai, and M. Zhao, "Impact of noise in a phytoplankton-zooplankton system," Journal of Applied Analysis \& Computation, vol. 10, no. 5, pp. 1878-1896, 2020.

[8] A. Mandal, P. K. Tiwari, S. Samanta, E. Venturino, and S. Pal, "A nonautonomous model for the effect of environmental toxins on plankton dynamics," Nonlinear Dynamics, vol. 99, no. 4, pp. 3373-3405, 2020.

[9] X. Y. Meng, N. N. Qin, and H. F. Huo, "Dynamics of a food chain model with two infected predators," International Journal of Bifurcation and Chaos, vol. 31, no. 2, Article ID 2150019, 2021.
[10] X. Y. Meng and J. Li, "Dynamical behavior of a delayed preypredator-scavenger system with fear effect and linear harvesting," International Journal of Biomathematics, vol. 14, no. 4, Article ID 2150024, 2021.

[11] J. Chattopadhyay, R. R. Sarkar, and S. Mandal, "Toxin-producing plankton may act as a biological control for planktonic blooms-field study and mathematical modelling," Journal of Theoretical Biology, vol. 215, no. 3, pp. 333-344, 2002.

[12] S. Chakraborty, S. Roy, and J. Chattopadhyay, "Nutrientlimited toxin production and the dynamics of two phytoplankton in culture media: a mathematical model," Ecological Modelling, vol. 213, no. 2, pp. 191-201, 2008.

[13] J. Chattopadhyay, R. R. Sarkar, and A. E. Abdllaoui, "A delay differential equation model on harmful algal blooms in the presence of toxic substances," Mathematical Medicine and Biology, vol. 19, no. 2, pp. 137-161, 2002.

[14] R. R. Sarkar, B. Mukhopadhyay, R. Bhattacharyya, and S. Banerjee, "Time lags can control algal bloom in two harmful phytoplankton-zooplankton system," Applied Mathematics and Computation, vol. 186, no. 1, pp. 445-459, 2007.

[15] T. Saha and M. Bandyopadhyay, "Dynamical analysis of toxin producing phytoplankton-zooplankton interactions," Nonlinear Analysis: Real World Applications, vol. 10, no. 1, pp. 314-332, 2009.

[16] Y. Wang, W. H. Jiang, and H. B. Wang, "Stability and global Hopf bifurcation in toxic phytoplankton-zooplankton model with delay and selective harvesting," Nonlinear Dynamics, vol. 73, no. 1-2, pp. 881-896, 2013.

[17] H. P. Zhu, W. Hui, Y. Z. Song, and J. Li, "Dynamical analysis of a toxin-producing phytoplankton-zooplankton model with refuge," Mathematical Biosciences and Engineering, vol. 14, no. 2, pp. 529-557, 2017.

[18] Z. Jiang, X. H. Bi, X. Bi, T. Zhang, and B. G. Sampath Aruna Pradeep, "Global Hopf bifurcation of a delayed phytoplankton-zooplankton system considering toxin producing effect and delay dependent coefficient," Mathematical Biosciences and Engineering, vol. 16, no. 5, pp. 3807-3829, 2019.

[19] J. G. Yang and S. L. Yuan, "Dynamics of a toxic producing phytoplankton-zooplankton model with three-dimensional patch," Applied Mathematics Letters, vol. 118, Article ID 107146, 2021.

[20] Y. Z. Pei, Y. F. Lv, and C. G. Li, "Evolutionary consequences of harvesting for a two-zooplankton one-phytoplankton system," Applied Mathematical Modelling, vol. 36, no. 4, pp. 1752-1765, 2012.

[21] Y. F. Lv, R. Yuan, and Y. Z. Pei, "Stable coexistence mediated by specialist harvesting in a two zooplankton-phytoplankton system," Applied Mathematical Modelling, vol. 37, no. 20-21, p. 9012, 2013.

[22] Y . F. Lv, J. Z. Cao, J. Song, R. Yuan, and Y. Z. Pei, “Global stability and Hopf-bifurcation in a zooplankton-phytoplankton model," Nonlinear Dynamics, vol. 76, no. 1, pp. 345-366, 2014.

[23] R. X. Shi and J. Yu, "Hopf bifurcation analysis of two zooplankton-phytoplankton model with two delays," Chaos, Solitons and Fractals, vol. 100, pp. 62-73, 2017.

[24] A. Mondal, A. K. Pal, and G. P. Samanta, "Rich dynamics of non-toxic phytoplankton, toxic phytoplankton and zooplankton system with multiple gestation delays," International Journal of Dynamics and Control, vol. 8, no. 1, pp. 112-131, 2020.

[25] J. R. Beddington, "Mutual interference between parasites or predators and its effect on searching efficiency," The Journal of Animal Ecology, vol. 44, no. 1, pp. 331-340, 1975. 
[26] D. L. DeAngelis, R. A. Goldstein, and R. V. O’Neill, “A model for tropic interaction," Ecology, vol. 56, no. 4, pp. 881-892, 1975.

[27] R. S. Cantrell and C. Cosner, "On the dynamics of predatorprey models with the Beddington-DeAngelis functional response," Journal of Mathematical Analysis and Applications, vol. 257, no. 1, pp. 206-222, 2001.

[28] R. Yuan and Z. H. Liu, "Stability and bifurcation in a delayed predator-prey system with Beddington-DeAngelis functional response," Journal of Mathematical Analysis and Application, vol. 296, no. 2, pp. 521-537, 2004.

[29] F. D. Chen and M. S. You, "Permanence, extinction and periodic solution of the predator-prey system with Beddington-DeAngelis functional response and stage structure for prey," Nonlinear Analysis: Real World Applications, vol. 9, no. 2, pp. 207-221, 2008.

[30] P. J. Pal and P. K. Mandal, "Bifurcation analysis of a modified Leslie-Gower predator-prey model with Beddington-DeAngelis functional response and strong Allee effect," Mathematics and Computers in Simulation, vol. 97, pp. 123-146, 2014.

[31] X. Y. Meng and J. G. Wang, "Analysis of a delayed diffusive model with Beddington-Deangelis functional response," International Journal of Biomathematics, vol. 12, no. 4, pp. 277-300, 2019.

[32] X. Y. Meng, Y. Q. Wu, and J. Li, "Dynamical analysis of a fuzzy phytoplankton-zooplankton model with refuge, fishery protection and harvesting," Journal of Applied Mathematics and Computing, vol. 63, no. 1-2, pp. 361-389, 2020.

[33] D. Y. Bai, J. S. Li, and W. R. Zeng, "Global stability of the boundary solution of a nonautonomous predator-prey system with Beddington-Deangelis functional response," Journal of Biological Dynamics, vol. 14, no. 1, pp. 421-437, 2020.

[34] A. C. Hardy, "Observations on the uneven distribution of oceanic plankton,” Discovery Reports, vol. 11, pp. 511-538, 1936.

[35] W. Z. Huang, "Global dynamics for a reaction-diffusion equation with time delay," Journal of Differential Equations, vol. 143 , no. 2 , pp. $293-326,1998$

[36] B. Mukhopadhyay and R. Bhattacharyya, "Modelling phytoplankton allelopathy in a nutrient-plankton model with spatial heterogeneity," Ecological Modelling, vol. 198, no. 1-2, pp. 163-173, 2006.

[37] J. T. Zhao and J. J. Wei, "Dynamics in a diffusive plankton system with delay and toxic substances effect," Nonlinear Analysis: Real World Applications, vol. 22, no. 1, pp. 66-83, 2015.

[38] R. J. Han and B. X. Dai, "Spatiotemporal dynamics and Hopf bifurcation in a delayed diffusive intraguild predation model with Holling II functional response," International Journal of Bifurcation and Chaos, vol. 26, no. 10, Article ID 1650197, 2016.

[39] Z. C. Jiang, W. Z. Zhang, J. Zhang, and T. Q. Zhang, "Dynamical analysis of a phytoplankton-zooplankton system with harvesting term and Holling III functional response," International Journal of Bifurcation and Chaos, vol. 28, no. 13, Article ID 1850162, 2018.

[40] R. J. Han and B. X. Dai, "Cross-diffusion induced Turing instability and amplitude equation for a toxic-phytoplanktonzooplankton model with nonmonotonic functional response," International Journal of Bifurcation and Chaos, vol. 27, no. 6, Article ID 1750088, 2017.

[41] W. Wang, S. T. Liu, and Z. B. Liu, "Spatiotemporal dynamics near the Turing-Hopf bifurcation in a toxic-phytoplankton- zooplankton model with cross-diffusion," Nonlinear Dynamics, vol. 98, no. 1, pp. 27-37, 2019.

[42] D. X. Jia, T. H. Zhang, and S. L. Yuan, "Pattern dynamics of a diffusive toxin producing phytoplankton-zooplankton model with three-dimensional patch," International Journal of Bifurcation and Chaos, vol. 29, no. 4, Article ID 1930011, 2019.

[43] R. J. Han and B. X. Dai, "Spatiotemporal pattern formation and selection induced by nonlinear cross-diffusion in a toxicphytoplankton-zooplankton model with Allee effect," Nonlinear Analysis: Real World Applications, vol. 45, pp. 822-853, 2019.

[44] R. Z. Yang and Y. T. Ding, "Spatiotemporal dynamics in a predator-prey model with a functional response increasing in both predator and prey densities," Journal of Applied Analysis and Computation, vol. 10, no. 5, pp. 1962-1979, 2020.

[45] X. Y. Meng and J. G. Wang, "Dynamical analysis of a delayed diffusive predator-prey model with schooling behaviour and Allee effect," Journal of Biological Dynamics, vol. 14, no. 1, pp. 826-848, 2020.

[46] K. J. Zhuang, G. Jia, and D. Z. Liu, "Stability and Hopf bifurcation in a three-component planktonic model with spatial diffusion and time delay," Complexity, vol. 2019, Article ID 4590915, 17 pages, 2019.

[47] F. Rao, "Spatiotemporal dynamics in a reaction-diffusion toxic-phytoplankton-zooplankton model," Journal of Statistical Mechanics Theory and Experiment, vol. 2013, no. 08, pp. 8014-8032, 2013.

[48] J. J. Anagnost and C. A. Desoer, "An elementary proof of the Routh-Hurwitz stability criterion," Circuits Systems and Signal Processing, vol. 10, no. 1, pp. 101-114, 1991.

[49] W. M. Liu, "Criterion of Hopf bifurcations without using eigenvalues," Journal of Mathematical Analysis and Applications, vol. 182, no. 1, pp. 250-256, 1994.

[50] Y. L. Song, M. A. Han, and J. J. Wei, "Stability and Hopf bifurcation analysis on a simplified BAM neural network with delays," Physica D: Nonlinear Phenomena, vol. 200, no. 3-4, pp. 185-200, 2005.

[51] B. D. Hassard, N. D. Kazarinoff, and Y. H. Wan, Theory and Applications of Hopf Bifurcation, Cambridge University: Cambridge University Press, Cambridge, UK, 1981.

[52] R. K. Gonshor, “A riesz representation theorem," Proceedings of the American Mathematical Society, vol. 24, no. 3, pp. 629-636, 1970.

[53] J. D. Murray, Mathematical Biology II: Spatial Models and Biomedical Applications, Springer-Verlag, Berlin, Germany, 2003.

[54] D. Henry, Geometric Theory of Semilinear Parabolic Equtions, Springer-Verlag, Berlin, Germany, 1981.

[55] J. H. Wu, Theory and Applications of Partial Functional Differential Equations, Springer, Berlin, Germany, 1996. 